\title{
Charge and spin density response functions of the clean two-dimensional electron gas with Rashba spin-orbit coupling at finite momenta and frequencies
}

\author{
M. Pletyukhov and S. Konschuh \\ Institut für Theoretische Festkörperphysik and Center for Functional Nanostructures, \\ Universität Karlsruhe, D-76128 Karlsruhe, Germany
}

\begin{abstract}
We analytically evaluate charge and spin density response functions of the clean two-dimensional electron gas with Rashba spin-orbit coupling at finite momenta and frequencies. On the basis of our exact expressions we discuss the accuracy of the long-wavelength and the quasiclassical approximations. We also derive the static limit of spin susceptibilities and demonstrate, in particular, how the Kohn-like anomalies in their derivatives are related to the spin-orbit modification of the Ruderman-Kittel-Kasuya-Yosida interaction. Taking into account screening and exchange effects of the Coulomb interaction, we describe the collective charge and spin density excitation modes which appear to be coupled due to nonvanishing spin-charge response function.

PACS numbers: 71.70.Ej,73.20.Mf, 73.21.-b
\end{abstract}

\section{INTRODUCTION}

One of the working principles of semiconductor spintronics 1.2 is based on the idea to exploit spin-orbit (SO) coupling for a manipulation of an electron's spin by means of electric fields. The SO coupling of the Rashba type $e^{\underline{3}}$ arises in a two-dimensional electron gas (2DEG) at semiconductor heterojunction due to the quantum well asymmetry in the perpendicular direction, and the strength $\alpha_{R}$ of this coupling can be tuned by a gate voltage $e^{4.5}$.

A theoretical description of SO-related phenomena in the 2DEG is provided by coupled transport equations for charge and spin components of the distribution function $\frac{6,7,8,9,10}{}$. In the regime of a linear response to external fields these equations appear to be intimately linked to the density response functions such as charge and spin susceptibilities and - more peculiar - spincharge response functions. In the presence of impurity scattering all of these functions have been previously evaluated in the quasiclassical approximation in the both diffusive $^{\underline{8}}\left(q \ll \frac{\hbar}{v_{F} \tau}\right)$ and non-diffusive $\underline{\underline{11}}\left(\frac{\hbar}{v_{F} \tau} \ll q \ll\right.$ $k_{F}$ ) regimes, where $q$ is a momentum transfer, $k_{F}$ and $v_{F}$ are Fermi momentum and Fermi velocity, and $\tau$ is an elastic scattering time.

Recently it has been also remarked ${ }^{11,12}$ that the quasiclassical results for the Rashba system are validated only in the presence of a finite amount of disorder such that $\tau^{-1} \gg m^{*} \alpha_{R}^{2}$, where $m^{*}$ is an effective electron's mass. Therefore, they cannot be straightforwardly applied in the extreme collisionless limit $\tau \rightarrow \infty$ even at small $q \ll k_{F}$, and the response functions of the clean 2DEG with Rashba SO coupling require a more refine consideration at finite values of $q$ and frequency $\omega$.

Various response functions in the clean case $\tau \rightarrow \infty$ are most easily evaluated in the long-wavelength limit $q \rightarrow 0^{6.13,14,15,16}$. On the other hand, the knowledge of the dynamic response functions at finite $q$ enables one to find dispersions of the collective charge and spin density excitations $17,18,19,20,21,22,23$ occurring in the presence of electron-electron interaction. In particular, in Ref. 23 the polarization operator, or the charge susceptibility, of the system in question has been calculated analytically at arbitrary momenta and frequencies, and the SO-induced attenuation of the charge density mode (plasmon) has been quantitatively described within the random phase approximation (RPA).

In this paper we analytically evaluate (Sec. III) the other density response functions following the computational scheme elaborated in Ref. 23. In Sec. IV we derive the static limit of the spin susceptibilities, and observe an occurrence of the Kohn-like anomalies ${ }^{24}$ in their derivatives. We demonstrate how they are related to the SO modification ${ }^{25}$ of the Ruderman-Kittel-KasuyaYosida (RKKY) interaction $26,27,28$ between two magnetic impurities. In Sec. $\mathrm{V}$ we compare our expressions for the dynamic response functions with the results of the long-wavelength and the quasiclassical approximations, and make conclusions about applicability ranges of the latter. Finally, in Sec. VI we revisit the problem of the collective charge and spin density excitations treating electron-electron interaction in terms of the Hubbard approximation ${ }^{29}$, i.e. our consideration extends beyond the RPA scheme. We demonstrate that the charge and the spin components are coupled in the obtained collective modes, which is a consequence of a simultaneous account of the non-zero spin-charge response function and the exchange vertex corrections.

\section{BASIC DEFINITIONS}

Let us consider the 2DEG with SO coupling of the Rashba type $\mathrm{3}^{3}$ which is described by the single-particle Hamiltonian

$$
H_{\mathbf{k}}=\frac{k^{2}}{2 m^{*}}+\alpha_{R} k h_{\mathbf{k}}^{R},
$$

where $h_{\mathbf{k}}^{R}=\sigma^{x} \sin \phi_{\mathbf{k}}-\sigma^{y} \cos \phi_{\mathbf{k}}$ is the spin-angular part of the Rashba spin-orbit coupling term, and we use the units such that $\hbar=1$. 
The spectrum of (1) is split into two subbands,

$$
\epsilon_{\mathbf{k}}^{ \pm}=\frac{k^{2}}{2 m^{*}} \pm \alpha_{\mathrm{R}} k
$$

the corresponding eigenstates being

$$
\psi_{\mathbf{k}+}=\frac{1}{\sqrt{2}}\left(\begin{array}{c}
1 \\
-i e^{i \phi_{\mathbf{k}}}
\end{array}\right), \quad \psi_{\mathbf{k}-}=\frac{1}{\sqrt{2}}\left(\begin{array}{c}
-i e^{-i \phi_{\mathbf{k}}} \\
1
\end{array}\right) .
$$

The matrix $\mathcal{U}_{\mathbf{k}}$ diagonalizing the initial Hamiltonian (1) as well as $h_{\mathbf{k}}^{R}$, i.e. $\sigma^{z}=\mathcal{U}_{\mathbf{k}}^{\dagger} h_{\mathbf{k}}^{R} \mathcal{U}_{\mathbf{k}}$, is then given by

$$
\mathcal{U}_{\mathbf{k}}=\left(\psi_{\mathbf{k}+} ; \psi_{\mathbf{k}-}\right)=\frac{1}{\sqrt{2}}\left[1-i \sigma^{x} \cos \phi_{\mathbf{k}}-i \sigma^{y} \sin \phi_{\mathbf{k}}\right] .
$$

It is also convenient to introduce the projectors onto the eigenstates (3),

$$
P_{\mathbf{k} \pm}=\psi_{\mathbf{k} \pm} \otimes \psi_{\mathbf{k} \pm}^{\dagger} \equiv \frac{1 \pm h_{\mathbf{k}}^{R}}{2}
$$

which allow us, for example, to spectrally decompose the Hamiltonian $H_{\mathbf{k}}=\sum_{\mu= \pm} \epsilon_{\mathbf{k}}^{\mu} P_{\mathbf{k} \mu}$ as well as the (retarded) Green's function

$$
G_{\mathbf{k} \epsilon}^{\mathrm{ret}}=\sum_{\mu= \pm} \frac{P_{\mathbf{k} \mu}}{\epsilon+i 0-\epsilon_{\mathbf{k}}^{\mu}} .
$$

A linear response $\rho_{\mathbf{q} \omega}^{\alpha}$ of the charge density $(\alpha=0)$ and the spin densities $(\alpha=x, y, z)$ to an external spatially inhomogeneous and nonstationary perturbation $V_{\mathbf{q} \omega}^{\beta}$, which consists of a scalar potential $(\beta=0)$ and a magnetic field $(\beta=x, y, z)$, is usually determined in the framework of the Kubo formalism ${ }^{29}$. Applying the standard technique of the linear response theory and using for convenience the representation (6), one can establish an expression for the (retarded) density-density response functions,

$$
\chi_{\mathbf{q} \omega}^{\alpha \beta}=\sum_{\mu, \mu^{\prime}= \pm} \int \frac{d^{2} \mathbf{k}}{(2 \pi)^{2}} \frac{n_{F}\left(\epsilon_{\mathbf{k}}^{\mu}\right)-n_{F}\left(\epsilon_{\mathbf{k}+\mathbf{q}}^{\mu^{\prime}}\right)}{\omega+i 0+\epsilon_{\mathbf{k}}^{\mu}-\epsilon_{\mathbf{k}+\mathbf{q}}^{\mu^{\prime}}} \mathcal{F}_{\mathbf{k}, \mathbf{k}+\mathbf{q} ; \mu, \mu^{\prime}}^{\alpha \beta},
$$

where

$$
\mathcal{F}_{\mathbf{k}, \mathbf{k}+\mathbf{q} ; \mu, \mu^{\prime}}^{\alpha \beta}=\operatorname{Tr}\left[P_{\mathbf{k} \mu} \sigma^{\alpha} P_{\mathbf{k}+\mathbf{q}, \mu^{\prime}} \sigma^{\beta}\right]
$$

are the overlap functions; $n_{F}$ denotes the Fermi distribution, and $\sigma^{0} \equiv 1$.

In the explicit form $\mathcal{F}_{\mathbf{k}, \mathbf{k}+\mathbf{q} ; \mu, \mu^{\prime}}^{\alpha \beta}$ are listed in Appendix B of Ref. 11. Here we quote their symmetry property, which can be directly established from the definition (8):

$$
\mathcal{F}_{\mathbf{k}, \mathbf{k}+\mathbf{q} ; \mu, \mu^{\prime}}^{\alpha \beta}=s \mathcal{F}_{-\mathbf{k}-\mathbf{q},-\mathbf{k} ; \mu^{\prime}, \mu}^{\alpha \beta},
$$

where $s=1$ for the charge-charge and spin-spin components, and $s=-1$ for the spin-charge components.

The expression (7) includes definitions of a polarization operator $(\alpha, \beta=0)$, spin susceptibilities $(\alpha, \beta=x, y, z)$ as well as of spin-charge response functions $(\alpha=0$ and $\beta=x, y, z$, or vice versa). In the presence of $\mathrm{SO}$ coupling the latter functions do not vanish, and their study represents an especial interest.

We note that the expression (77) can be alternatively found in terms of the equations of motion for the local charge and spin densities (see Appendix $\mathrm{A}$ for details). A matrix formulation of this approach provides a convenient tool for an account of screening and exchange effects in presence of electron-electron interaction. In more detail this will be discussed in Sec. VI.

\section{EVALUATION OF $\chi^{\alpha \beta}$}

The functions (7) have been previously treated at finite $q$ in terms of different approximations. The most typical of them are: 1) the small- $q$ (long-wavelength) formal expansion of the whole integrand (see, e.g., Refs. 6] 13); and 2) the quasiclassical approximation (see, e.g., Ref. 17) which is usually performed in the quantum kinetic equation approach.

In Ref. 23 the polarization operator $\chi^{00}$ in the clean limit has been evaluated beyond these approximations, and the obtained result has been used for an estimation of the accuracy of the long-wavelength expansion. In particular, the latter has been shown to be applicable in the limited range of the very small $q \ll k_{R}^{2} / k_{F}$, where $k_{F}=\sqrt{2 m^{*} \epsilon_{F}+k_{R}^{2}}$ and $k_{R}=m^{*} \alpha_{R}$ is the Rashba momentum splitting.

As for the quasiclassical approximation, it has been argued in Ref. 11 that its application in the presence of SO coupling is validated at the finite values of the disorder broadening $\tau^{-1} \gg k_{R}^{2} / m^{*}$, which smoothens the divergences of the quasiclassical result near the boundaries of the SO-induced (intersubband) particle-hole excitation region in the $(q, \omega)$-plane. One still might hope that the quasiclassical approximation is trustful in the extreme collisionless limit $\tau \rightarrow \infty$, provided one does not come too close to the boundaries in question. For this reason we are going to revisit its accuracy in the context of our present calculations.

It also remains unclear how the two above mentioned approximations are related to each other in the clean limit. Both of them are elaborated for small values of $q$, but seem to give different results even at $q \rightarrow 0$. For example, an application of the long-wavelength expansion to the optical conductivity yields a box-like function 13,14 which is finite at frequencies $2 \alpha_{R} k_{F}-2 m^{*} \alpha_{R}^{2}<\omega<$ $2 \alpha_{R} k_{F}+2 m^{*} \alpha_{R}^{2}$, while in the quasiclassical approximation the width of this frequency window cannot be resolved at all.

In this section we are going to evaluate the functions (7) without making any kind of approximations. We will neatly follow the computational scheme elaborated in Ref. 23 for $\chi^{00}$. Thus, we will derive analytic expressions for the other response functions and discuss their limiting behavior in the subsequent sections. 
We start from the observation that, due to the momentum space isotropy of the spectrum (2), the response functions (7) can be represented in the form

$$
\begin{aligned}
& \chi_{\mathbf{q} \omega}^{\alpha \beta}=\sum_{\mu, \lambda= \pm} \chi_{\mathbf{q} \omega, \lambda}^{\alpha \beta, \mu}, \\
& \chi_{\mathbf{q} \omega, \lambda}^{\alpha \beta, \mu}=\int \frac{d^{2} \mathbf{k}}{(2 \pi)^{2}} n_{F}\left(\epsilon_{\mathbf{k}}^{\mu}\right) \lambda^{\frac{1-s}{2}} \\
& \times\left[\frac{\mathcal{F}_{\mathbf{k}, \mathbf{k}+\mathbf{q} ; \mu, \mu}^{\alpha \beta}}{\epsilon_{\mathbf{k}}^{\mu}-\epsilon_{\mathbf{k}+\mathbf{q}}^{\mu}+\lambda(\omega+i 0)}+\frac{\mathcal{F}_{\mathbf{k}, \mathbf{k}+\mathbf{q} ; \mu,-\mu}^{\alpha \beta}}{\epsilon_{\mathbf{k}}^{\mu}-\epsilon_{\mathbf{k}+\mathbf{q}}^{-\mu}+\lambda(\omega+i 0)}\right]
\end{aligned}
$$

where the factor $\lambda^{\frac{1-s}{2}}$ originates from the property (9). It is convenient to choose the basis in the momentum space such that $\mathbf{q}$ is aligned with $x$-direction. Then the matrix $\chi$ becomes sparse, the non-vanishing terms being $\chi^{00}, \chi^{x x}, \chi^{y y}, \chi^{z z}, \chi^{0 y}=\chi^{y 0}$, and $\chi^{z x}=-\chi^{x z}$. Introducing $x=\cos \left(\phi_{\mathbf{k}}-\phi_{\mathbf{q}}\right) \equiv \cos \phi$, we obtain the following expressions at zero temperature

$$
\begin{aligned}
& \left(\begin{array}{c}
\chi_{\mathbf{q} \omega, \lambda}^{00, \mu} \\
\chi_{\mathbf{q} \omega, \lambda}, \lambda
\end{array}\right)=\frac{1}{8 \pi^{2}} \int_{0}^{k_{F}-\mu k_{R}} k d k \int_{0}^{2 \pi} d \phi \\
& \times\left[\frac{1 \pm \frac{k+q x}{|\mathbf{k}+\mathbf{q}|}}{\epsilon_{\mathbf{k}}^{\mu}-\epsilon_{\mathbf{k}+\mathbf{q}}^{\mu}+\lambda(\omega+i 0)}+\frac{1 \mp \frac{k+q x}{|\mathbf{k}+\mathbf{q}|}}{\epsilon_{\mathbf{k}}^{\mu}-\epsilon_{\mathbf{k}+\mathbf{q}}^{-\mu}+\lambda(\omega+i 0)}\right], \\
& \left(\begin{array}{c}
\chi_{\mathbf{q} \omega, \lambda}^{y y, \mu} \\
\chi_{\mathbf{q} \omega, \lambda}^{x \omega, \mu}
\end{array}\right)=\frac{1}{8 \pi^{2}} \int_{0}^{k_{F}-\mu k_{R}} k d k \int_{0}^{2 \pi} d \phi \\
& \times\left[\frac{1 \pm \frac{k\left(2 x^{2}-1\right)+q x}{|\mathbf{k}+\mathbf{q}|}}{\epsilon_{\mathbf{k}}^{\mu}-\epsilon_{\mathbf{k}+\mathbf{q}}^{\mu}+\lambda(\omega+i 0)}+\frac{1 \mp \frac{k\left(2 x^{2}-1\right)+q x}{|\mathbf{k}+\mathbf{q}|}}{\epsilon_{\mathbf{k}}^{\mu}-\epsilon_{\mathbf{k}+\mathbf{q}}^{-\mu}+\lambda(\omega+i 0)}\right], \\
& \left(\begin{array}{c}
\chi_{\mathbf{q} \omega, \lambda}^{0 y, \mu} \\
i \chi_{\mathbf{q} \omega, \lambda}^{2 x, \lambda}
\end{array}\right)=\frac{\mu(-\lambda)^{\frac{1 \pm 1}{2}}}{8 \pi^{2}} \int_{0}^{k_{F}-\mu k_{R}} k d k \int_{0}^{2 \pi} d \phi \\
& \times\left[\frac{x \pm \frac{q+k x}{|\mathbf{k}+\mathbf{q}|}}{\epsilon_{\mathbf{k}}^{\mu}-\epsilon_{\mathbf{k}+\mathbf{q}}^{\mu}+\lambda(\omega+i 0)}+\frac{x \mp \frac{q+k x}{|\mathbf{k}+\mathbf{q}|}}{\epsilon_{\mathbf{k}}^{\mu}-\epsilon_{\mathbf{k}+\mathbf{q}}^{-\mu}+\lambda(\omega+i 0)}\right] .
\end{aligned}
$$

Note that the components of $\chi$ in the arbitrary basis can be recovered by an orthogonal rotation in the $x-y$ plane (see Appendix B of Ref. 11).

After simple algebra we eliminate the odd powers of $|\mathbf{k}+\mathbf{q}|$ in Eqs. (12)-(14). It means that the corresponding integrands happen to be rational functions of $k$ and $\cos \phi$. Let us also note the identity

$$
\chi^{x x}+\chi^{y y}=\chi^{00}+\chi^{z z}
$$

which allows us to express, say, $\chi^{x x}$ in terms of the other diagonal components. There remain, in fact, only five independent functions $\chi^{(j)}=\left\{\chi^{00}, \chi^{0 y}, \chi^{y y}, \chi^{z z}, i \chi^{z x}\right\}$, which can be conveniently labeled by the index $j=$ $1, \ldots, 5$. Like in Ref. 23, we also introduce the index $i=1,2,3,4$ which denotes different combinations of $\{\mu, \lambda\}=\{-,+\},\{+,+\},\{-,-\},\{+,-\}$, respectively.
Defining the dimensionless units $y=k_{R} / k_{F}, z=$ $q / 2 k_{F}, v=k / k_{F}$, and $w=m^{*} \omega / 2 k_{F}^{2}$, we cast (12)-(14) into the form

$$
\begin{aligned}
-\frac{1}{\nu} \operatorname{Im} \chi_{i}^{(j)} & =\int_{0}^{1-\mu y} v g_{i}^{(j)}(v, z, w, y) d v \\
-\frac{1}{\nu} \operatorname{Re} \chi_{i}^{(j)} & =\check{f}_{i}^{(j)}+\int_{0}^{1-\mu y} v f_{i}^{(j)}(v, z, w, y) d v
\end{aligned}
$$

where $\nu \equiv \nu_{2 D}=\frac{m^{*}}{2 \pi}$ is the density of states in 2DEG per each spin component. The functions $g_{i}^{(j)}$ and $f_{i}^{(j)}$ are given by

$$
\begin{aligned}
g_{i}^{(j)}= & \frac{\lambda C_{i}^{(j)}}{2} \int_{0}^{2 \pi} d \phi \operatorname{sign}\left(2 v z x-\mu y v+2\left(z^{2}-\lambda w\right)\right) \\
& \times\left(x+\delta_{i}^{(j)}\right) \delta\left(x^{2}+\beta_{i} x+\gamma_{i}\right) \\
f_{i}^{(j)}= & \frac{C_{i}^{(j)}}{2 \pi} \int_{0}^{2 \pi} d \phi \frac{x+\delta_{i}^{(j)}}{x^{2}+\beta_{i} x+\gamma_{i}}
\end{aligned}
$$

where the coefficients

$$
\begin{aligned}
\beta_{i} & =\frac{2\left(z^{2}-\lambda w\right)-\mu y(v+\mu y)}{v z} \\
\gamma_{i} & =\frac{\left(z^{2}-\lambda w\right)^{2}-\mu y v\left(z^{2}-\lambda w\right)-z^{2} y^{2}}{v^{2} z^{2}}
\end{aligned}
$$

are the same for each $j$, and $\delta(\ldots)$ in (18) denotes the Dirac delta function. The difference between the response functions $\chi^{(j)}$ appears only in the form of the coefficients $\check{f}_{i}^{(j)}, C_{i}^{(j)}$, and $\delta_{i}^{(j)}$, which are listed in Appendix B for all $j$ 's.

We note that the real part of $\chi^{(j)}$ can be represented as a sum

$$
\operatorname{Re} \chi^{(j)}=\check{\chi}^{(j)}+\operatorname{Re} \chi^{(j), I}+\operatorname{Re} \chi^{(j), I I}
$$

where the term $\check{\chi}^{(j)}=-\nu \sum_{i} \check{f}_{i}^{(j)}$ is nonzero only for $j=3$ and $j=5$ [see Eq. (B11)]. The terms $\operatorname{Re} \chi^{(j), I}$ and $\operatorname{Re} \chi^{(j), I I}$ are obtained by integrating the functions $\sum_{i} v f_{i}^{(j), I}$ and $\sum_{i} v f_{i}^{(j), I I}$, where $f_{i}^{(j), I}=f_{i}^{(j)} \Theta\left(\beta_{i}^{2}-4 \gamma_{i}\right)$ and $f_{i}^{(j), I I}=f_{i}^{(j)} \Theta\left(4 \gamma_{i}-\beta_{i}^{2}\right)$.

Performing angular integration and a subsequent change of the variable $v \rightarrow \tau(v)$ according to Eq. (34) of Ref. 23, we obtain expressions for $\operatorname{Im} \chi^{(j)}$ and $\operatorname{Re} \chi^{(j), I}$ in the form of $\chi^{(1)}$ found previously,

$$
\begin{gathered}
-\frac{1}{\nu} \operatorname{Im} \chi^{(j)}=\sum_{\sigma, \mu= \pm} \sigma \int_{\tau_{\sigma+}(y)}^{\tau_{\sigma+}(\mu)} d \tau \mathcal{L}^{(j)+}(\tau) \\
+\Theta(1-4 w) \sum_{\mu= \pm} \int_{\tau_{+-}(\mu)}^{\tau_{--}(\mu)} d \tau \mathcal{L}^{(j)-}(\tau) \\
+2 \Theta\left(y^{2}-4 w\right) \int_{\tau_{--}(y)}^{\tau_{+-}(y)} d \tau \mathcal{L}^{(j)-}(\tau),
\end{gathered}
$$


and

$$
\begin{aligned}
& -\frac{1}{\nu} \operatorname{Re} \chi^{(j), I}=\sum_{\sigma, \mu= \pm} \int_{\tau_{\sigma+}(y)}^{\tau_{\sigma+}(\mu)} d \tau \mathcal{R}^{(j)+}(\tau) \\
& +\Theta(1-4 w) \sum_{\sigma, \mu= \pm} \int_{-\mu \tau_{++}(0)}^{\tau_{\sigma-}(\mu)} d \tau \mathcal{R}^{(j)-}(\tau) \\
& +2 \Theta\left(y^{2}-4 w\right) \sum_{\sigma= \pm} \int_{\tau_{\sigma-}(y)}^{\tau_{-+}(0)} d \tau \mathcal{R}^{(j)-}(\tau),
\end{aligned}
$$

where

$$
\begin{aligned}
\tau_{1,2} & = \pm w / z, \quad \tau_{3,4}=-y \pm z \\
\tau_{\sigma \lambda}(x) & =\frac{1}{2}\left[-x+\sigma \sqrt{x^{2}+4 \lambda w}\right] \\
\mathcal{L}^{(j) \pm}(\tau) & =\mathcal{L}^{(j)}(\tau) \operatorname{sign}\left(\tau^{2}+y \tau \pm w\right) \\
\mathcal{R}^{(j) \pm}(\tau) & =\mathcal{R}^{(j)}(\tau) \operatorname{sign}(\tau z \mp w(\tau+y) / z) .
\end{aligned}
$$

In the representation (23)-(24) the actual integration limits are universal for all response function. The difference appears only in the form of the integrands

$$
\begin{aligned}
\mathcal{L}^{(j)}(\tau) & =Q^{(j)}(\tau) \frac{\Theta(P(\tau))}{\sqrt{P(\tau)}}, \\
\mathcal{R}^{(j)}(\tau) & =Q^{(j)}(\tau) \frac{\Theta(-P(\tau))}{\sqrt{-P(\tau)}}, \\
P(\tau) & =\prod_{k=1}^{4}\left(\tau-\tau_{k}\right),
\end{aligned}
$$

which are specified for each response function by

$$
\begin{aligned}
Q^{(1)}(\tau) & =\frac{1}{2 z}\left(\tau-\tau_{3}\right)\left(\tau-\tau_{4}\right), \\
Q^{(2)}(\tau) & =-\frac{w}{z \tau} Q^{(1)}(\tau), \\
Q^{(3)}(\tau) & =\frac{w^{2}}{z^{2} \tau^{2}} Q^{(1)}(\tau), \\
Q^{(4)}(\tau) & =\frac{z}{2 \tau^{2}}\left(\tau-\tau_{1}\right)\left(\tau-\tau_{2}\right), \\
Q^{(5)}(\tau) & =\frac{\tau+y}{z} Q^{(4)}(\tau) .
\end{aligned}
$$

Interestingly, for the function $\chi^{x x}=\chi^{00}-\chi^{y y}+\chi^{z z}$ one would obtain the term

$$
Q^{(1)}(\tau)-Q^{(3)}(\tau)+Q^{(4)}(\tau)=\frac{(\tau+y)^{2}}{z^{2}} Q^{(4)}(\tau),
$$

which is anticipated after comparison of Eqs. (35) and (36) with (32)-(34).

Let us make several comments about the obtained results (23) and (24).

1) First of all, note that the overall sign in the second line of (23) differs from its counterpart in the corresponding equation (35) of Ref. 23. We use the present opportunity to correct the misprint in the previously derived expression. Fortunately, it did not affect any other result of Ref. 23 .

2) The actual intervals of integration in Eq. (23) are explicitly written down in Eqs. (B23)-(B26). None of them contains the point $\tau=0$, which means that one should not worry about the convergence of integrals over $\mathcal{L}^{(3,4,5)}(\tau) \sim 1 / \tau^{2}$ near this point.

3) The explicit analytic relations for $\operatorname{Im} \chi^{(j)}$ in terms of elliptic functions ${ }^{30}$ can be found for all $j$ 's in the same fashion as it has been done before for $\chi^{(1)}$ [see Appendix C of Ref. 23].

4) Some of the actual integration intervals in Eq. (24) do contain the point $\tau=0$, which means that the corresponding integrals $\int d \tau \mathcal{R}^{(3,4,5)}(\tau)$ are divergent in its vicinity. However, the whole expression Eq. (24) is convergent and well-defined, since the singularities exactly cancel each other. In order to make Eq. (24) practically useful, one has to substitute $\int d \tau \mathcal{R}^{(j)}(\tau)$ by the corresponding difference of primitives, which can be also found in terms of elliptic functions.

5) Eq. (24) contains only the contribution $\operatorname{Re} \chi^{(j), I}$ to the full function $\operatorname{Re} \chi^{(j)}$. The contribution $\check{\chi}^{(j)}$ is quoted in (B11), and it remains to calculate the contribution $\operatorname{Re} \chi^{(j), I I}$. Making a complex change of variables $\tau=\frac{1}{2}\left[-\mu(v+\mu y)+i \sqrt{4 w-(v+\mu y)^{2}}\right]$ (cf. Eq. (34) of Ref. 23) one can as well find $\operatorname{Re} \chi^{(j), I I}$ in the analytic form which would involve the same integrand $\mathcal{R}^{(j)}(\tau)$ and a path of integration lying in the complex $\tau$-plane. Omitting technical details of this evaluation, we present the explicit expressions for $\operatorname{Re} \chi^{(j), I}+\operatorname{Re} \chi^{(j), I I}$ in Eqs. (B29)(B32).

\section{STATIC LIMIT}

In order to find a static limit of $\chi^{\alpha \beta}$ one should consider with caution Eqs. (B29) and (B32) at $w \rightarrow 0$. One can then find

$$
\begin{aligned}
- & \nu^{-1} \lim _{w \rightarrow 0} \chi^{00}=2+\frac{\pi}{2} \Theta(y-|z-1|) \sin \psi \\
- & \sum_{\mu= \pm} \Theta(z-(1-\mu y))\left(\mu \psi_{\mu} \sin \psi+\cos \psi_{\mu}+2 l_{\mu} \cos \psi\right) \\
& -2 \Theta(z-1) \cos \psi \operatorname{arccosh} z, \\
- & \nu^{-1} \lim _{w \rightarrow 0} \chi^{z z}=2+\frac{2}{\cos \psi} \sum_{\mu= \pm} \Theta(z-(1-\mu y)) l_{\mu} \\
& +\frac{2 \Theta(z-1)}{\cos \psi}\left(\operatorname{arccosh} z-\sqrt{1-(1 / z)^{2}}\right), \\
- & \nu^{-1} \lim _{w \rightarrow 0} \chi^{y y}=2-2 \Theta(z-1) \cos \psi \sqrt{1-(1 / z)^{2}},(40) \\
- & i \nu^{-1} \lim _{w \rightarrow 0} \chi^{z x}=\frac{\pi}{2} \Theta(y-|z-1|) \\
& -\sum_{\mu= \pm} \Theta(z-(1-\mu y))\left(\mu \psi_{\mu}-2 l_{\mu} \tan \psi\right) \\
& +2 \Theta(z-1) \tan \psi\left(\operatorname{arccosh} z-\sqrt{1-(1 / z)^{2}}\right),
\end{aligned}
$$




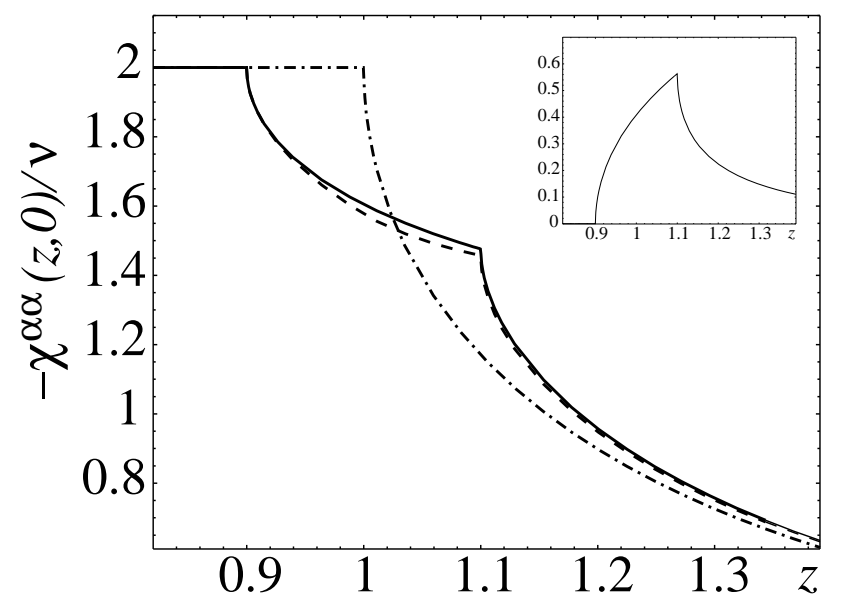

FIG. 1: Diagonal components of the static spin susceptibility near $z=q / 2 k_{F} \sim 1$ plotted at $y=k_{R} / k_{F}=0.1$. Solid, dashed-dotted, and dashed lines correspond to $\alpha=x, \alpha=y$, and $\alpha=z$, respectively. The inset shows the off-diagonal component $-i \chi^{z x}(z, 0) / \nu$.

where $\sin \psi=y / z($ for $y<z), \sin \psi_{\mu}=(1-\mu y) / z$ (for $1-\mu y<z$; note that this notation differs from its counterpart in Ref. 23 by $\mu \rightarrow-\mu$ ), and

$$
l_{\mu}=l_{\mu}(z)=\ln \frac{1+z \sin \left(\psi_{\mu}+\mu \psi\right)}{2 \sqrt{2 z} \cos \frac{1}{2} \psi_{\mu} \cos \frac{1}{2} \psi} .
$$

The static limit of $\chi^{x x}$ can be found from the identity (15). The off-diagonal spin-charge term $\chi^{0 y}$ identically vanishes in this limit, which means a decoupling of charge and spin components at zero frequency.

On the basis of the derived expressions (38)-(41) one can observe that for $z \leq 1-y$ all diagonal terms are equal to $\chi^{\alpha \alpha}=-2 \nu$, while their large- $z$ asymptotes are $\chi^{\alpha \alpha} \approx$ $-\nu \frac{1+y^{2}}{z^{2}}$. The off-diagonal spin-spin term $\chi^{z x}$ equals zero at $z \leq 1-y$, and $\chi^{z x} \approx \frac{2 i \nu y}{z^{3}}\left(1+\frac{2 y^{2}}{3}\right)$ at large $z \gg$ 1. The behavior of all components of the static spin susceptibility near $z \sim 1$ is shown in Fig. 1. One can see that $\chi^{y y}$ has a discontinuous derivative at $z=1$, while the derivatives of $\chi^{x x}, \chi^{z z}$, and $\chi^{z x}$ are discontinuous at $z=1 \mp y$. These anomalies are analogous to the Kohn anomaly of the polarization operator ${ }^{23,24,29}$ at $z=1$.

Using (39)-(41) we can find a SO-modification of the Ruderman-Kittel-Kasuya-Yosida (RKKY) Hamiltonian 26,27,28, which describes an indirect exchange interaction between two localized magnetic impurities. In general case the RKKY Hamiltonian reads 25

$$
H_{1,2}^{R K K Y}=J_{R K K Y}^{2} \sum_{\alpha, \beta=x, y, z} S_{1}^{\alpha} \chi^{\alpha \beta}\left(\mathbf{r}_{12}\right) S_{2}^{\beta},
$$

where $\mathbf{S}_{1,2}$ are the spin operators of impurities, and $\mathbf{r}_{12}=$ $\mathbf{r}_{1}-\mathbf{r}_{2}$ is the distance between them.

In the presence of SO coupling the Hamiltonian (43) becomes anisotropic in spin space, since the matrix $\chi$ is no longer proportional to the unit matrix. Let us find asymptotic values of $\chi^{\alpha \beta}\left(\mathbf{r}_{12}\right)$ at large $r_{12} \gg k_{F}^{-1}$. For simplicity we assume that the vector $\mathbf{r}_{12}$ is aligned with $x$-direction in the coordinate space. Inspecting (39)(41) and restoring the dimensional units, we establish the asymptotic form of the right-sided derivatives

$$
\begin{aligned}
&\left.\frac{d \chi^{y y}}{d q}\right|_{q \rightarrow q_{c 0}^{+}} \approx \frac{\nu}{2 k_{F}} \sqrt{\frac{2 q_{c 0}}{q-q_{c 0}}}, \\
&\left.\left.\frac{d \chi^{z z}}{d q}\right|_{q \rightarrow q_{c \mu}^{+}} \approx \frac{d \chi^{x x}}{d q}\right|_{q \rightarrow q_{c \mu}^{+}} \approx \frac{\nu}{4 k_{F}} \sqrt{\frac{2 q_{c \mu}}{q-q_{c \mu}}}, \\
&\left.\frac{d \chi^{z x}}{d q}\right|_{q \rightarrow q_{c \mu}^{+}} \approx \frac{i \mu \nu}{4 k_{F}} \sqrt{\frac{2 q_{c \mu}}{q-q_{c \mu}}}
\end{aligned}
$$

near the discontinuity points $q_{c 0}=2 k_{F}$ and $q_{c \mu}=2 k_{F}-$ $2 \mu k_{R}$. Performing the Fourier transformation of $\chi^{\alpha \beta}(q)$, we obtain the following leading asymptotic terms

$$
\begin{aligned}
\chi^{y y}\left(r_{12}\right) & \approx-\frac{\nu}{\pi} \frac{\sin \left(2 k_{F} r_{12}\right)}{r_{12}^{2}} \\
\chi^{z z}\left(r_{12}\right) & \approx \chi^{x x}\left(r_{12}\right) \approx-\frac{\nu}{2 \pi} \sum_{\mu= \pm} \frac{q_{c \mu} \sin \left(q_{c \mu} r_{12}\right)}{2 k_{F} r_{12}^{2}} \\
& \approx-\frac{\nu}{\pi} \frac{\sin \left(2 k_{F} r_{12}\right)}{r_{12}^{2}} \cos \left(2 k_{R} r_{12}\right) \\
\chi^{z x}\left(r_{12}\right) & \approx-\frac{\nu}{2 \pi} \sum_{\mu= \pm} \mu \frac{q_{c \mu} \cos \left(q_{c \mu} r_{12}\right)}{2 k_{F} r_{12}^{2}} \\
& \approx-\frac{\nu}{\pi} \frac{\sin \left(2 k_{F} r_{12}\right)}{r_{12}^{2}} \sin \left(2 k_{R} r_{12}\right)
\end{aligned}
$$

which oscillate in the coordinate space with the periods $2 \pi / q_{c 0}$ and $2 \pi / q_{c \mu}$. Substituting them into Eq. (43), we obtain

$$
H_{1,2}^{R K K Y}=F_{2}\left(r_{12}\right) \sum_{\alpha, \beta=x, y, z} S_{1}^{\alpha} O^{\alpha \beta}\left(\theta_{12}\right) S_{2}^{\beta},
$$

where the range function

$$
F_{2}\left(r_{12}\right)=-J_{R K K Y}^{2} \frac{\nu}{\pi} \frac{\sin \left(2 k_{F} r_{12}\right)}{r_{12}^{2}}
$$

is the same as in the absence of SO coupling. The SOmodification of the Hamiltonian (43) consists in the spin twist determined by the orthogonal transformation

$$
O\left(\theta_{12}\right)=\left(\begin{array}{ccc}
\cos \theta_{12} & 0 & -\sin \theta_{12} \\
0 & 1 & 0 \\
\sin \theta_{12} & 0 & \cos \theta_{12}
\end{array}\right)
$$

with the rotation angle $\theta_{12}=2 k_{R} r_{12}$. The expression (50) is in agreement with the corresponding result of Ref. 25. 


\section{BEHAVIOR OF THE RESPONSE FUNCTIONS $\chi^{\alpha \beta}$ AT SMALL MOMENTA}

\section{A. Exact expressions for $\operatorname{Im} \chi^{\alpha \beta}$ in the SO-induced particle-hole excitation region}

It has been discussed in Ref. 23 that an account of the Rashba SO coupling leads to an extension of the boundaries of a particle-hole continuum, or Landau damping region, which is defined by $\operatorname{Im} \chi^{(1)} \neq 0$. It has been also established that this extension has a shape of the wedge bounded by the parabolas $-(z-y)^{2}-(z-y) \equiv w_{4}(z)<$ $w<w_{1}(z) \equiv(z+y)^{2}+(z+y)$ [see Fig. 2 .

Since the representation (12)-(14) manifests the same pole structure for all response functions, their imaginary parts appear to be nonzero in the same domain where $\operatorname{Im} \chi^{(1)} \neq 0$. Analyzing (B25), we extract an explicit expression for $\operatorname{Im} \chi^{(j)}$ in the SO-induced particle-hole excitation region

$$
\begin{aligned}
- & \frac{1}{\nu} \operatorname{Im} \chi^{(j)}=-\Theta\left(w_{2}-w\right) \Theta\left(w-w_{4}\right) A_{2 c}^{(j)}(z-y) \\
& -\Theta\left(w_{1}-w\right) \Theta\left(w-w_{2}\right) A_{2 c}^{(j)}\left(-t_{1}\right) \\
& +\Theta\left(w_{3}-w\right) \Theta\left(w-w_{4}\right) A_{2 c}^{(j)}\left(-t_{3}\right)
\end{aligned}
$$

where $w_{1,2}=(z \pm y)^{2} \pm(z \pm y)$ and $w_{3,4}=-(z \pm y)^{2} \pm(z \pm$ $y)$. The functions $A_{2 c}^{(j)}$ and the arguments $t_{1}$ and $t_{3}$ are defined in Eqs. (B17), (B27) and (B28), respectively. On the analogy of $A_{2 c}^{(1)}$ explicitly quoted in Ref. 23 , one can as well express the rest $A_{2 c}^{(j)}$ in terms of elliptic functions.

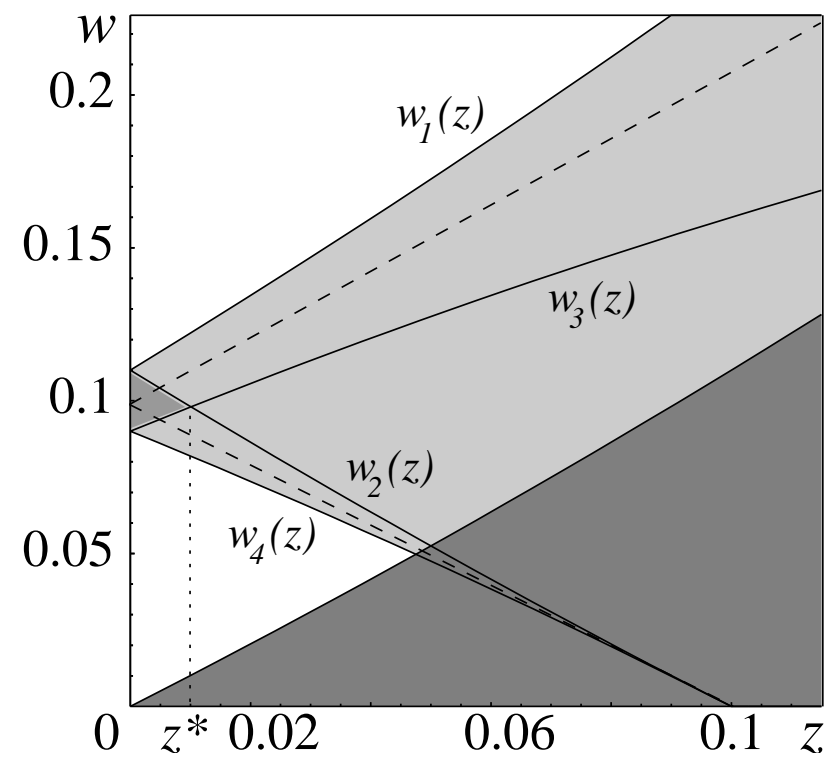

FIG. 2: SO-induced extension of the particle-hole excitation region at $y=0.1$ - the light-gray area bounded by the parabolas $w_{1} \equiv w_{+}$and $w_{4} \equiv w_{-}$. The small darkened triangle inside it indicates the range of applicability of the long-wavelength approximation. The dashed lines depict quasiclassical boundaries $w_{ \pm}^{q c}=y \pm z$.

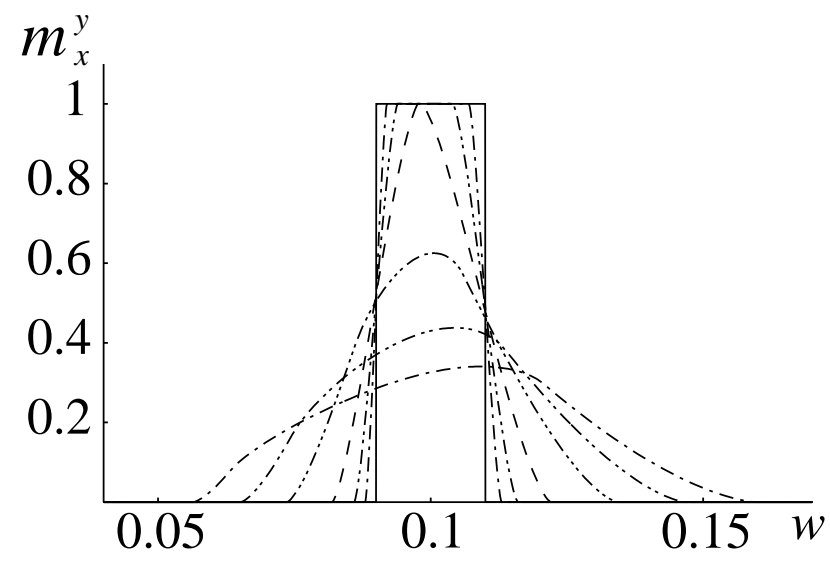

FIG. 3: Spin-galvanic function (54) at $y=0.1$ and the values of $z=0.0,0.025,0.005,0.01,0.02,0.03,0.04$. In particular, the solid line depicts the long-wavelength limit, and the dashed curve corresponds to $z=y^{2}=0.01 \approx z^{*}$.

For example, in Eqs. (B18) and (B19) we write down explicit formulas for $A_{2 c}^{(2)}$ and $A_{2 c}^{(4)}$, respectively.

Eq. (53) allows us to study the behavior of the density-density response functions at finite momenta. On its basis we can also describe the spin-density response $\rho^{y}=2 M_{x}^{y} E_{x}$ to a longitudinal electric field $E_{x}=-e \frac{\partial V^{0}}{\partial x}$ (or $E_{x}=-i q V^{0}$ in the momentum representation). The spin-galvanic response function ${ }^{31,32,33} M_{x}^{y}$ is then related to $\chi^{0 y}$ via $M_{x}^{y}=i e \chi^{0 y} /(2 q)$. Let us also introduce the rescaled function

$$
m_{x}^{y}=-\frac{16 k_{R}}{e \pi \nu} \operatorname{Re} M_{x}^{y} \equiv \frac{4 y}{\pi z \nu} \operatorname{Im} \chi^{0 y} .
$$

Using (B18), we calculate and plot it in Fig. 3 at fixed finite values of $z$. We remark that its frequency dependence in the range $w_{4}<w<w_{1}$ corresponding to intersubband transitions is similar to that of the finite- $q$ conductivity studied in Ref. 23 .

\section{B. Long-wavelength limit}

Spatially uniform spin susceptibilities of the 2DEG with SO coupling have been previously considered in Ref. 15. We recover the corresponding expressions in the long-wavelength limit

$$
-\lim _{z \rightarrow 0} \frac{\chi^{z z}}{\nu}=-2 \lim _{z \rightarrow 0} \frac{\chi^{x x(y y)}}{\nu}=2+\frac{w}{2 y^{2}} r(w),
$$

where

$$
r(w)=\ln \left|\frac{\left(w-y^{2}\right)^{2}-y^{2}}{\left(w+y^{2}\right)^{2}-y^{2}}\right|+i \pi \Theta\left(y^{2}-|w-y|\right) .
$$

The small- $q$ behavior of the polarization operator of 2DEG with Rashba SO coupling has been approximated 
in Refs. 6,13 by the expression

$$
-\frac{\chi^{00}}{\nu} \approx-\frac{z^{2}}{w^{2}}+\frac{z^{2}}{4 w} r(w),
$$

which is obtained after the formal expansion of $\chi^{00}$ in a series of $z=\frac{q}{2 k_{F}} \ll 1$. Later on it has been remarked ${ }^{23}$ that the formula (57) is, in fact, reliable only for the values $z<z^{*} \approx y^{2}$, where $z^{*}$ is the point of intersection of the parabolas $w_{2}(z)$ and $w_{3}(z)$ [see Fig. 2. Analogously, we can find approximate relations for the off-diagonal terms

$$
-\frac{i \chi^{z x}}{\nu} \approx \frac{z w}{2 y^{3}}\left[r(w)+\sum_{\mu= \pm} \frac{w y(\mu-2 y)}{y^{2}(1-\mu y)^{2}-w^{2}}\right]
$$

and

$$
\frac{\chi^{0 y}}{\nu} \approx \frac{z}{4 y}\left[r(w)+\frac{4 y^{2}}{w}\right],
$$

which are also applicable in the small triangular region located at $z<z^{*}$ and bounded by $w_{3}<w<w_{2}$. In particular, Eq. (59) accounts for the box-like shape of the function $m_{x}^{y}$ (54) in the linit $z \rightarrow 0$ which can be seen in Fig. 3,

\section{Quasiclassical approximation}

On the basis of the exact result (53) we can also estimate how accurate the quasiclassical approximation appears to be, when it is applied to a description of the clean 2DEG with Rashba SO coupling.

The quasiclassical approximation relies on the fact that all energy scales in the system are much smaller than the Fermi energy: $q k_{F} / m^{*}, \omega, \alpha_{R} k_{F} \ll \epsilon_{F}$. This inequality enables one to treat (12)-(14) linearizing the branches of the spectrum $\epsilon_{\mathbf{k}}^{\mu}$ near the corresponding Fermi points $k_{\mu}=k_{F}-\mu k_{R}$ and expanding $\mathcal{F}_{\mathbf{k}, \mathbf{k}+\mathbf{q} ; \mu, \mu^{\prime}}^{\alpha \beta}$ in a series of $q$. We note that a more systematic procedure of making the quasiclassical approximation is based on the gradient expansion of the quantum kinetic equation ${ }^{6,7,9}$, which is applicable in more general - nonequilibrium - situations.

Quasiclassical response functions $\chi^{\alpha \beta}$ of the disordered 2DEG with Rashba SO coupling have been explicitly calculated in Ref. 11. It has been also argued therein that the quasiclassical approximation is justified in the presence of a finite amount of impurities such that $\tau^{-1}>k_{R}^{2} / m^{*}$. This condition confines $\tau^{-1}$ from below. Had we ignored it, we would have obtained in the extreme collisionless limit $\tau \rightarrow \infty$ the quasiclassical expression for, say, out-of-plane component of the spin susceptibility in the form

$$
-\frac{1}{\nu} \operatorname{Im} \chi_{q c}^{z z}=w \sum_{\mu= \pm} \frac{\Theta\left(z^{2}-(w-\mu y)^{2}\right)}{\sqrt{z^{2}-(w-\mu y)^{2}}},
$$

which is divergent near $w=w_{ \pm}^{q c} \equiv|y \pm z|$.

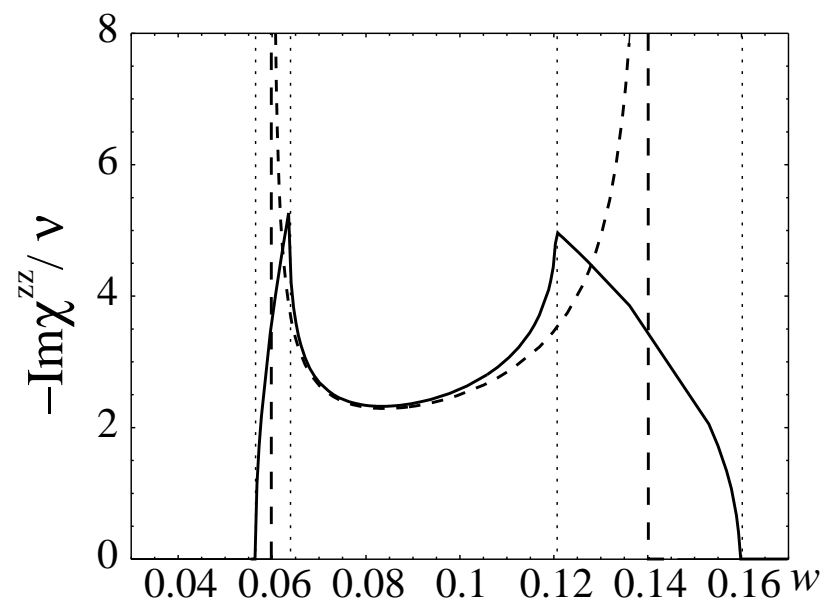

FIG. 4: Comparison of the exact (solid line) and the quasiclassical (dashed line) functions $-\operatorname{Im} \chi^{z z} / \nu$ at $z=0.04$ and $y=0.1$. Vertical dotted lines correspond (from the left to the right) to the frequency values $w_{4}, w_{2}, w_{3}$, and $w_{1}$. Vertical dashed lines correspond to the values $w_{-}^{q c}=y-z$ and $w_{+}^{q c}=y+z$ demarcating the quasiclassical boundaries of the SO-induced particle-hole excitation region.

The lines $w_{ \pm}^{q c}$ represent the quasiclassical boundaries of the SO-induced (intersubband) particle-hole excitation region, and they differ from the actual parabolic ones $w_{+} \equiv w_{1}$ and $w_{-} \equiv w_{4}$. In Fig. $22 w_{ \pm}^{q c}$ are depicted by the dashed lines, and one can observe that $w_{+}^{q c}$ lies in between $w_{1}$ and $w_{3}$, and $w_{-}^{q c}$ lies in between $w_{2}$ and $w_{4}$. It is also evident that in the quasiclassical approximation the finite basis $y-y^{2}<w<y+y^{2}$ of the wedge at $z=0$ is not resolved. This means that the imaginary part of a quasiclassical counterpart of $r(w)$ (56) appears to be delta-peaked, and therefore the corresponding finitevalued results of Sec. $\mathrm{VB}$ can not be reproduced in the quasiclassical approximation.

It can be anticipated that the quasiclassical approximation is still reliable in the triangular area $w_{2}<w<w_{3}$

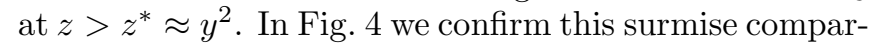
ing the function (60) with the exact expression calculated on the basis of Eqs. (53) and (B19).

Thus, we conclude that the long-wavelength and the quasiclassical approximations do not have any correspondence between each other, since they are applicable in the domains which do not overlap, i.e. at $z<z^{*}$ and $z>z^{*}$, respectively.

\section{COLLECTIVE CHARGE AND SPIN DENSITY EXCITATIONS}

Let us now consider the renormalization of the matrix $\chi$ due to electron-electron interaction. Treating the latter in the Hubbard approximation ${ }^{29}$, we take into account screening and exchange effects. An effective response matrix $\widetilde{\chi}$ [see Eq. A26)] is derived in Appendix A using the method of the equations of motion 29 . 
In the basis specified by the condition $\mathbf{q} \| \mathbf{e}_{x}$ the matrix $\chi$ can be decomposed into two $2 \times 2$-blocks

$$
\chi_{(0 y)}=\left(\begin{array}{cc}
\chi^{00} & \chi^{0 y} \\
\chi^{y 0} & \chi^{y y}
\end{array}\right), \quad \chi_{(x z)}=\left(\begin{array}{ll}
\chi^{x x} & \chi^{x z} \\
\chi^{z x} & \chi^{z z}
\end{array}\right) .
$$

It follows from (A26) that the spin-charge $\chi_{(0 y)}$ and the spin-spin $\chi_{(x z)}$ blocks are renormalized independently of each other. In particular,

$$
\begin{aligned}
& \tilde{\chi}_{(0 y)}=\left(1-\chi_{(0 y)} F_{(0 y)}\right)^{-1} \chi_{(0 y)}, \\
& \tilde{\chi}_{(x z)}=\left(1+J \chi_{(x z)}\right)^{-1} \chi_{(x z)},
\end{aligned}
$$

where $F_{(0 y)}=\operatorname{diag}\left\{v_{q}-J,-J\right\} \approx \operatorname{diag}\left\{v_{q},-J\right\}, v_{q}=$ $\frac{2 \pi e^{2}}{q}$ is the Coulomb interaction, and $J=\frac{\pi e^{2}}{k_{F}}$ is the Hubbard's vertex exchange term at small $q \ll k_{F}$.

Let us first consider the spin-charge block and find the renormalized response functions

$$
\begin{aligned}
& \tilde{\chi}^{00}=\frac{\chi^{00}+J \bar{\Delta}_{(0 y)}}{\Delta_{(0 y)}}, \\
& \tilde{\chi}^{y y}=\frac{\chi^{y y}-v_{q} \bar{\Delta}_{(0 y)}}{\Delta_{(0 y)}}, \\
& \tilde{\chi}^{0 y}=\frac{\chi^{0 y}}{\Delta_{(0 y)}},
\end{aligned}
$$

where

$$
\begin{aligned}
\bar{\Delta}_{(0 y)} & =\operatorname{det} \chi_{(0 y)}=\chi^{00} \chi^{y y}-\left(\chi^{0 y}\right)^{2}, \\
\Delta_{(0 y)} & =\operatorname{det}\left(1-\chi_{(0 y)} F_{(0 y)}\right) \\
& \approx\left(1-v_{q} \chi^{00}\right)\left(1+J \chi^{y y}\right)+J v_{q}\left(\chi^{0 y}\right)^{2} .
\end{aligned}
$$

For the spin-spin block we have

$$
\begin{aligned}
& \tilde{\chi}^{x x}=\frac{\chi^{x x}+J \bar{\Delta}_{(z x)}}{\Delta_{(z x)}}, \\
& \tilde{\chi}^{z z}=\frac{\chi^{z z}+J \bar{\Delta}_{(z x)}}{\Delta_{(z x)}}, \\
& \tilde{\chi}^{x z}=\frac{\chi^{x z}}{\Delta_{(x z)}},
\end{aligned}
$$

where

$$
\begin{aligned}
\bar{\Delta}_{(x z)} & =\operatorname{det} \chi_{(z x)}=\chi^{x x} \chi^{z z}+\left(\chi^{x z}\right)^{2} \\
\Delta_{(x z)} & =\operatorname{det}\left(1+J \chi_{(x z)}\right) \\
& =\left(1+J \chi^{x x}\right)\left(1+J \chi^{z z}\right)+J^{2}\left(\chi^{x z}\right)^{2} .
\end{aligned}
$$

Dispersions of the collective charge and spin density excitations are determined from the equations $\Delta_{(0 y)}=0$ and $\Delta_{(x z)}=0$, and the response functions $\widetilde{\chi}^{\alpha \beta}$ are strongly enhanced at the parameter values satisfying these conditions.

Before quantifying $\widetilde{\chi}^{\alpha \beta}$, let us qualitatively discuss the role of the spin-charge mixing term $\chi^{0 y}$ as well as the role of the exchange corrections. Note that if either $\chi^{0 y}=0$ or

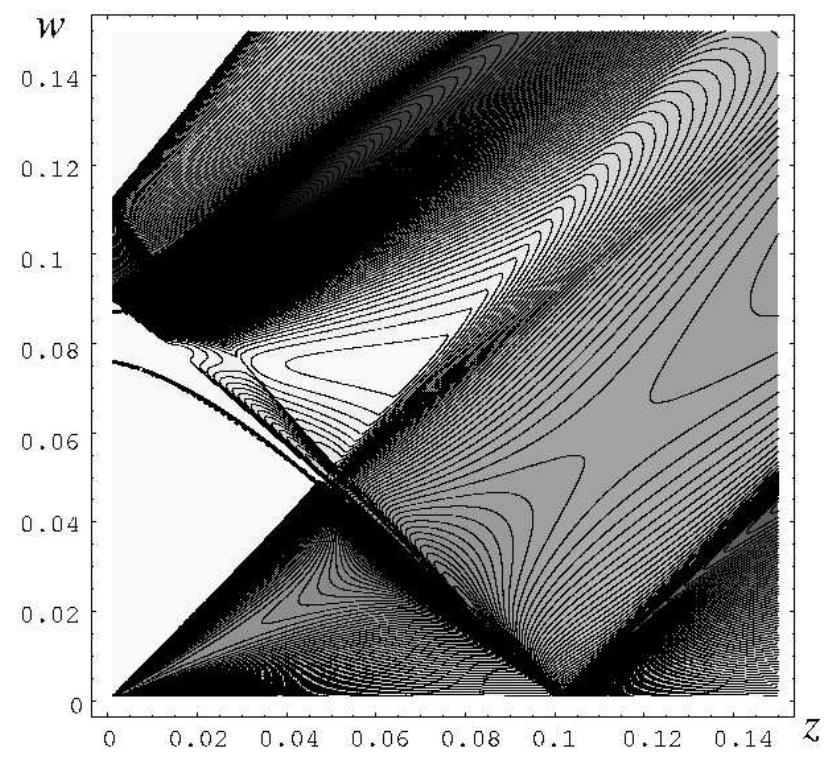

FIG. 5: Contour plot of $-\operatorname{Im} \tilde{\chi}^{x x}(z, w) / \nu$. Parameters: $y=$ 0.1 and $r_{s}=0.6$.

$J=0$, the dynamics of the charge and the spin- $y$ densities becomes decoupled, and the corresponding plasmon and $\mathrm{SDE}_{y}$ (spin- $y$ density excitation) modes are independently found from the conditions $1-v_{q} \chi^{00}=0$ and $1+J \chi^{y y}=0$ (the latter equation makes sense at $J \neq 0$ only). The role of $\chi^{0 y}$, whatever small it might be, is considerably strengthened when the dispersions of the plasmon and the $\mathrm{SDE}_{y}$ modes come close to a degeneracy point. In fact, $\chi^{0 y} \neq 0$ lifts this degeneracy, thus making a possible crossing of these modes avoidable. Therefore, we expect that an account of the spin-charge mixing along with the exchange interaction would lead to nontrivial features in profiles of $\widetilde{\chi}^{00}$ and $\widetilde{\chi}^{y y}$ near the avoided intersection. It is also implied that the peaks corresponding to the both collective modes would appear in every response function of the spin-charge block. In particular, the spin susceptibility $\tilde{\chi}^{y y}$ is expected to manifest resonant features at the position of the plasmon dispersion, while $\widetilde{\chi}^{00}$ should have a peak corresponding to the $\operatorname{SDE}_{y}$ mode.

We remark that the decoupled plasmon mode in 2DEG with Rashba SO coupling has been previously considered in Refs. $19,20,21,23$ in the random phase approximation (RPA), i.e. at $J=0$. In turn, the coupled spin$x-\operatorname{spin}-z$ collective modes, which are determined by $\Delta_{(x z)}=0$, occur at $J \neq 0$, i.e. their description requires an extension of the RPA. Their dispersions, as well as the dispersion of the decoupled spin- $y$ mode (at neglected spin-charge mixing $\chi^{0 y}=0$ ), have been previously considered in Ref. 17 in terms of the Hubbard's approximation with the bare spin susceptibilities calculated in the quasiclassical approximation (see Sec. $\mathrm{VC}$ ).

Using our exact expressions for $\chi^{\alpha \beta}$, we are able to study dispersions of the collective charge and spin density modes in more detail. They can be visualized, for 


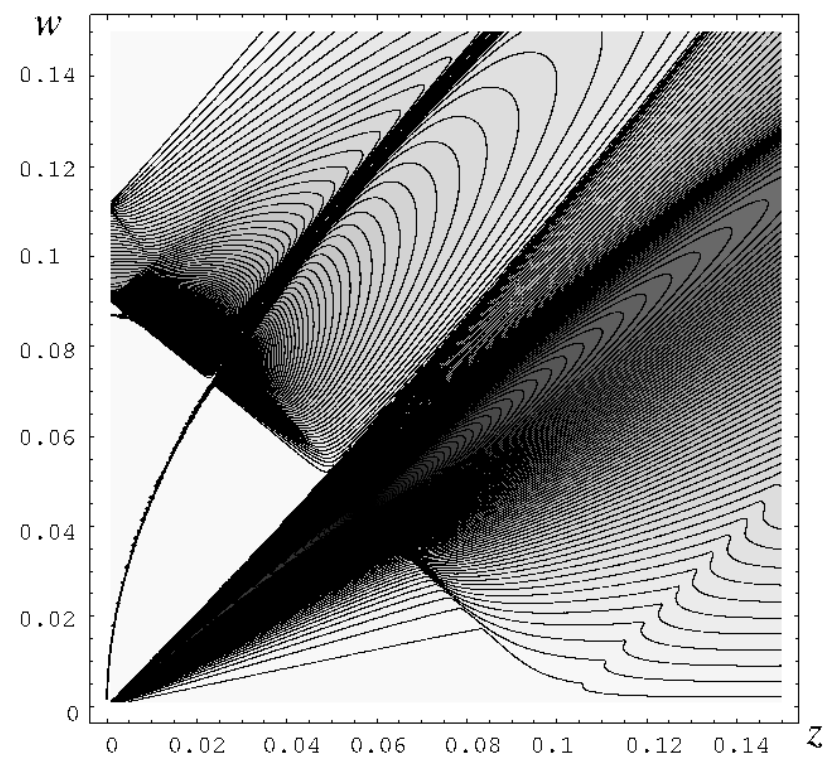

FIG. 6: Contour plot of $-\operatorname{Im} \tilde{\chi}^{y y}(z, w) / \nu$. Parameters: $y=$ 0.1 and $r_{s}=0.6$.

example, in contour plots of the response functions $\widetilde{\chi}^{\alpha \beta}$. Absolute values of $\widetilde{\chi}^{\alpha \beta}$ providing a useful information for inelastic Raman scattering 34 are available as well.

Let us start our consideration from the renormalized response functions (69)-(71) constituting the spin-spin block $\tilde{\chi}_{(x z)}$. In Fig. 5] we present the contour plot of $-\operatorname{Im} \tilde{\chi}^{x x} / \nu$ at $y=0.1$ and the Wigner-Seitz parameter $r_{s} \equiv \frac{\sqrt{2} m^{*} e^{2}}{k_{F}}=0.6$ (note that $J=\frac{r_{s}}{2 \sqrt{2} \nu}$ ). One can observe the dispersions of the two coupled spin- $x-\operatorname{spin}-z$ collective modes. We state that their spectra are in the qualitative agreement with those previously predicted in Ref. 17 on the basis of the quasiclassical approximation. However, the absolute values of $\widetilde{\chi}^{\alpha \beta}$ differ from their quasiclassical counterparts $\tilde{\chi}_{q c}^{\alpha \beta}$. The reason is the same as discussed in Sec. $\mathrm{VC}$ for the case of bare susceptiblities.

In turn, the response functions (64)- 66 of the spincharge block $\tilde{\chi}_{(0 y)}$ manifest novel qualitative features due to the account of the spin-charge mixing $\chi^{0 y}$ along with the exchange interaction. In Fig. 6] we present the contour plot of the function $-\operatorname{Im} \tilde{\chi}^{y y} / \nu$ at $y=0.1$ and $r_{s}=0.6$ (note that $v_{q}=\frac{r_{s}}{2 \sqrt{2} z \nu}$ ). It also contains the two collective modes: plasmon-like and $\mathrm{SDE}_{y}$-like. The plasmon-like mode has almost the same dispersion $w \approx \sqrt{\frac{r_{s} z}{2 \sqrt{2}}}$ as the plasmon mode in the absence of SO coupling. The $\mathrm{SDE}_{y}$-like mode originates at $z=0$ from the finite frequency value slightly below the bottom of the wedge. Being undamped, these modes do not come close to each other. They collide soon after the plasmonlike mode enters into the SO-induced damping region, i.e. at $z \geq 0.023$. In the vicinity of this point the spincharge mixing $\chi^{0 y}$ acquires its importance. Although a pictorial description of the avoided crossing loses its obviousness because of the modes' broadening, we prove

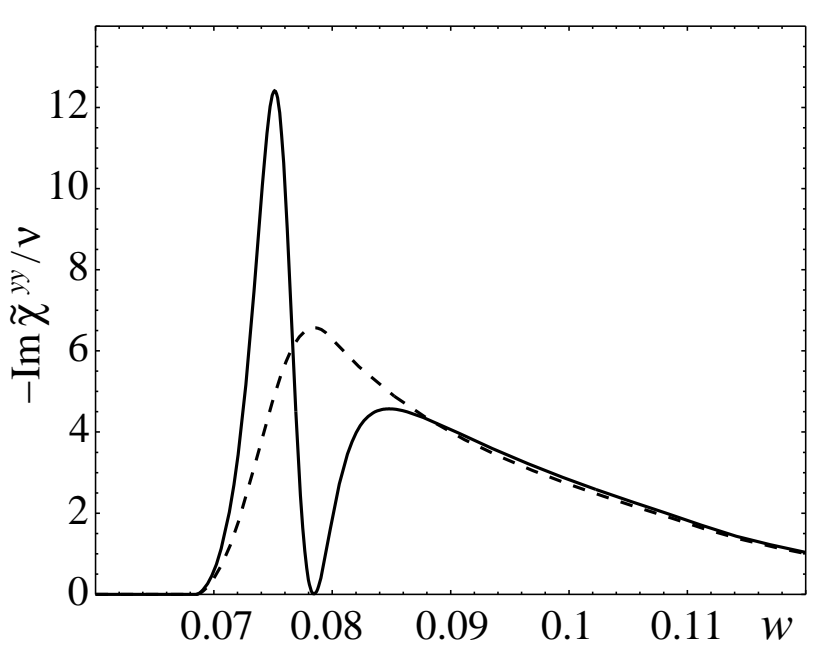

FIG. 7: The cross section of $-\operatorname{Im} \tilde{\chi}^{y y}(z, w) / \nu$ at $z=0.027$ (solid line). The dashed line corresponds to the case of intentionally neglected spin-charge mixing term $\chi^{0 y}$. Parameters: $y=0.1$ and $r_{s}=0.6$.

that it does happen. For this purpose we plot in Fig. 7 the cross-section of $-\operatorname{Im} \widetilde{\chi}^{y y} / \nu$ at $z=0.027$. Thereby we show that instead of the only $\mathrm{SDE}_{y}$ peak (dashed line) occurring at intentionally neglected $\chi^{0 y}=0$, we obtain at $\chi^{0 y} \neq 0$ the two well-resolved peaks (solid line) corresponding to the plasmon-like and the $\mathrm{SDE}_{y}$-like modes. A formation of the dip between them is interpreted as an avoided crossing of the two broadened modes. A relevance of such interpretation becomes even more evident, if one would compare several subsequent cross sections of $-\operatorname{Im} \tilde{\chi}^{y y} / \nu$ and $-\operatorname{Im} \tilde{\chi}^{00} / \nu$.

As one can see from Eq. (66), the spin-charge mixing term is renormalized by electron-electron interaction as

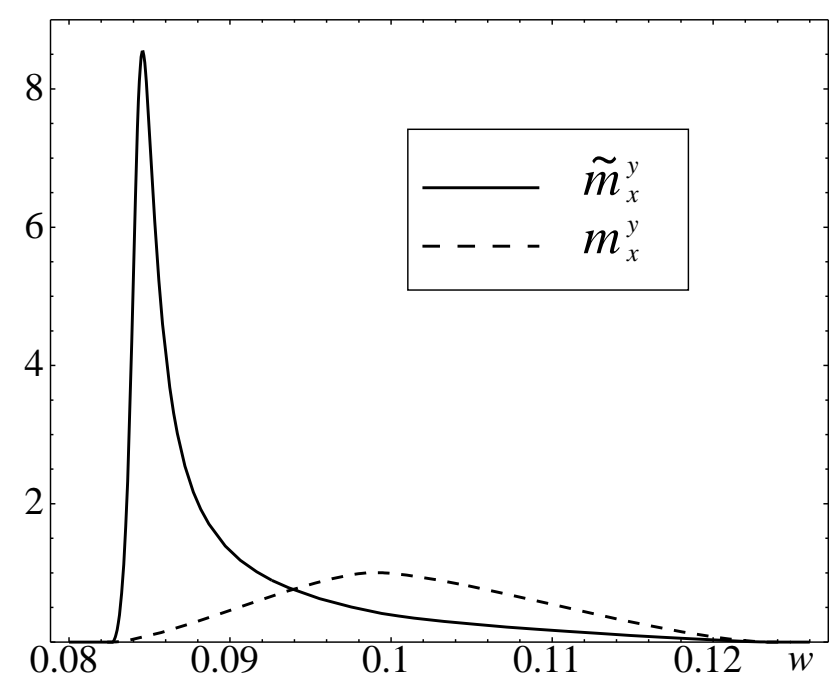

FIG. 8: The renormalized spin-galvanic function (74) at $z=$ 0.01 and the parameters $y=0.1$ and $r_{s}=0.6$ (solid line). The dashed line depicts its non-interacting counterpart $\left(r_{s}=0\right)$ given by (54). 
well. Let us define the function

$$
\widetilde{m}_{x}^{y}=\frac{4 y}{\pi z \nu} \operatorname{Im} \tilde{\chi}^{0 y},
$$

which is an interacting counterpart of the bare spingalvanic function $m_{x}^{y}$ (54). In Fig. 8 we compare both of them at $z=0.01$ and $y=0.1$ in the frequency window $y-2 y^{2}<w<y+2 y^{2}$. We observe that at these parameters the major effect of renormalization consists in a considerable amplification of the spin-galvanic function (74) due to the exchange interaction.

\section{SUMMARY}

We have derived zero-temperature analytic expressions for the charge and spin density response functions of the clean 2DEG with Rashba SO coupling at finite momenta and frequencies. We have studied their static and longwavelength limits as well as established the applicability range of the quasiclassical approximation. In the static limit we have observed the Kohn-like anomalies in the spin susceptibilities and showed how they are related to the SO modification of the RKKY interaction.

The renormalization of the response functions due to electron-electron interaction has been considered in the Hubbard's approximation. We have studied the collective charge and spin density modes which appear to be coupled due to the nonvanishing spin-charge mixing term $\chi^{0 y}$. One of the important consequences of this coupling is the emergence of the plasmon-like peak in the spectrum of the renormalized spin susceptibility.

\section{ACKNOWLEDGEMENTS}

We are grateful to Gerd Schön for useful discussions. M.P. acknowledges the financial support from the Deutsche Forschungsgemeinschaft (DFG).

\section{APPENDIX A: EQUATIONS OF MOTION}

Let us define the local charge $(\alpha=0)$ and $\operatorname{spin}(\alpha=$ $x, y, z)$ density operators

$$
\hat{\rho}_{\mathbf{q}}^{\alpha}=\sum_{\mathbf{k}} \hat{\rho}_{\mathbf{k q}}^{\alpha}, \quad \hat{\rho}_{\mathbf{k q}}^{\alpha}=c_{\mathbf{k}}^{\dagger} \sigma^{\alpha} c_{\mathbf{k}+\mathbf{q}}
$$

and derive the equations of motion 29 for the expectation values $\rho_{\mathbf{k q}}^{\alpha}=\left\langle\hat{\rho}_{\mathbf{k q}}^{\alpha}\right\rangle$. In the Fourier representation they read

$$
\omega \rho_{\mathbf{k q}}^{\alpha}=\left\langle\left[\hat{\rho}_{\mathbf{k q}}^{\alpha}, H\right]\right\rangle .
$$

The full Hamiltonian $H=H_{0}+H_{\text {ext }}$ consists of the parts describing a system

$$
H_{0}=\sum_{\mathbf{k}^{\prime}, \beta} E_{\mathbf{k}^{\prime}}^{\beta} \hat{\rho}_{\mathbf{k}^{\prime}, 0}^{\beta}
$$

and an external perturbation

$$
H_{\mathrm{ext}}=\frac{1}{\mathcal{V}} \sum_{\mathbf{q}^{\prime}, \beta} V_{\mathbf{q}^{\prime}}^{\beta} \hat{\rho}_{-\mathbf{q}^{\prime}}^{\beta}
$$

where $\mathcal{V}$ is the spatial volume. For the Rashba system (1) we have $E_{\mathbf{k}}^{\beta}=\left(\epsilon_{\mathbf{k}}, \alpha_{R} k_{y},-\alpha_{R} k_{x}, 0\right)$. Later on we will also add the interaction term $H_{\text {int }}$ to the Hamiltonian $H$.

Using the identity

$$
\left[\hat{\rho}_{\mathbf{k q}}^{\alpha}, \hat{\rho}_{\mathbf{k}^{\prime}, 0}^{\beta}\right]=c_{\mathbf{k}}^{\dagger} \sigma^{\alpha} \sigma^{\beta} c_{\mathbf{k}^{\prime}} \delta_{\mathbf{k}+\mathbf{q}, \mathbf{k}^{\prime}}-c_{\mathbf{k}^{\prime}}^{\dagger} \sigma^{\beta} \sigma^{\alpha} c_{\mathbf{k}+\mathbf{q}} \delta_{\mathbf{k k}^{\prime}}(
$$

we find

$$
\begin{aligned}
\left\langle\left[\hat{\rho}_{\mathbf{k q}}^{\alpha}, H_{0}\right]\right\rangle & =\sum_{\beta} E_{\mathbf{k}+\mathbf{q}}^{\beta}\left\langle c_{\mathbf{k}}^{\dagger} \sigma^{\alpha} \sigma^{\beta} c_{\mathbf{k}+\mathbf{q}}\right\rangle \\
& -\sum_{\beta} E_{\mathbf{k}}^{\beta}\left\langle c_{\mathbf{k}}^{\dagger} \sigma^{\beta} \sigma^{\alpha} c_{\mathbf{k}+\mathbf{q}}\right\rangle .
\end{aligned}
$$

It is convenient to introduce the representation

$$
\omega \rho_{\mathbf{k q}}^{\alpha}-\left\langle\left[\hat{\rho}_{\mathbf{k q}}^{\alpha}, H_{0}\right]\right\rangle=\sum_{\beta} \mathcal{A}_{\mathbf{k q}}^{\alpha \beta} \rho_{\mathbf{k q}}^{\beta}
$$

in terms of the matrix $\mathcal{A}$ with the elements

$$
\begin{aligned}
& \mathcal{A}_{\mathbf{k q}}^{\alpha \alpha}=\omega+\epsilon_{\mathbf{k}}-\epsilon_{\mathbf{k}+\mathbf{q}}, \\
& \mathcal{A}_{\mathbf{k q}}^{x 0}=\mathcal{A}_{\mathbf{k q}}^{0 x}=-\alpha_{R} q_{y}, \\
& \mathcal{A}_{\mathbf{k q}}^{y 0}=\mathcal{A}_{\mathbf{k q}}^{0 y}=\alpha_{R} q_{x}, \\
& \mathcal{A}_{\mathbf{k q}}^{x z}=-\mathcal{A}_{\mathbf{k q}}^{z x}=i \alpha_{R}\left(2 k_{x}+q_{x}\right), \\
& \mathcal{A}_{\mathbf{k q}}^{y z}=-\mathcal{A}_{\mathbf{k q}}^{z y}=i \alpha_{R}\left(2 k_{y}+q_{y}\right), \\
& \mathcal{A}_{\mathbf{k q}}^{0 z}=\mathcal{A}_{\mathbf{k q}}^{z 0}=\mathcal{A}_{\mathbf{k q}}^{x y}=\mathcal{A}_{\mathbf{k q}}^{y x}=0 .
\end{aligned}
$$

In order to find the commutator of $\hat{\rho}_{\mathbf{k q}}^{\alpha}$ with $H_{\text {ext }}$ (A4), we use the identity

$$
\left[\hat{\rho}_{\mathbf{k q}}^{\alpha}, \hat{\rho}_{-\mathbf{q}^{\prime}}^{\beta}\right]=c_{\mathbf{k}}^{\dagger} \sigma^{\alpha} \sigma^{\beta} c_{\mathbf{k}+\mathbf{q}-\mathbf{q}^{\prime}}-c_{\mathbf{k}+\mathbf{q}^{\prime}}^{\dagger} \sigma^{\beta} \sigma^{\alpha} c_{\mathbf{k}+\mathbf{q}} .
$$

From the whole sum over $\mathbf{q}^{\prime}$ in (A4) we pick out the only term $\mathbf{q}^{\prime}=\mathbf{q}$. This is known as the random phase approximation (RPA). Then,

$$
\left[\hat{\rho}_{\mathbf{k q}}^{\alpha}, H_{\mathrm{ext}}\right] \approx \frac{1}{\mathcal{V}} \sum_{\beta} V_{\mathbf{q}}^{\beta}\left(c_{\mathbf{k}}^{\dagger} \sigma^{\alpha} \sigma^{\beta} c_{\mathbf{k}}-c_{\mathbf{k}+\mathbf{q}}^{\dagger} \sigma^{\beta} \sigma^{\alpha} c_{\mathbf{k}+\mathbf{q}}\right),
$$

and after averaging we obtain

$$
\left\langle\left[\hat{\rho}_{\mathbf{k q}}^{\alpha}, H_{\mathrm{ext}}\right]\right\rangle \approx \frac{1}{\mathcal{V}} \sum_{\beta} \mathcal{B}_{\mathbf{k q}}^{\alpha \beta} V_{\mathbf{q}}^{\beta},
$$

where

$$
\mathcal{B}_{\mathbf{k q}}^{\alpha \beta}=\left\langle c_{\mathbf{k}}^{\dagger} \sigma^{\alpha} \sigma^{\beta} c_{\mathbf{k}}-c_{\mathbf{k}+\mathbf{q}}^{\dagger} \sigma^{\beta} \sigma^{\alpha} c_{\mathbf{k}+\mathbf{q}}\right\rangle .
$$

In order to fulfil the averaging, we have to transform $c_{\mathbf{k}}=\mathcal{U}_{\mathbf{k}} \gamma_{\mathbf{k}}$ into the diagonal basis $\gamma_{\mathbf{k} \pm}$ such that

$$
\left\langle\gamma_{\mathbf{k} \mu}^{\dagger} \gamma_{\mathbf{k} \mu^{\prime}}\right\rangle=\delta_{\mu \mu^{\prime}} f_{\mathbf{k}}^{\mu}
$$


The transformation matrix $\mathcal{U}_{\mathbf{k}}$ is defined in Eq. (4). Since the external perturbation is assumed to be small, we average in (A13) with respect to the system's density matrix. Therefore we can identify $f_{\mathbf{k}}^{\mu} \equiv n_{F}\left(\epsilon_{\mathbf{k}}^{\mu}\right)$.

The components (A12) are then found to be

$$
\begin{aligned}
\mathcal{B}_{\mathbf{k q}}^{\alpha \beta} & =\frac{1}{2} \sum_{\mu} f_{\mathbf{k}}^{\mu} \operatorname{Tr}\left[\left(1+\mu \sigma^{z}\right) \mathcal{U}_{\mathbf{k}}^{\dagger} \sigma^{\alpha} \sigma^{\beta} \mathcal{U}_{\mathbf{k}}\right] \\
& -\frac{1}{2} \sum_{\mu} f_{\mathbf{k}+\mathbf{q}}^{\mu} \operatorname{Tr}\left[\left(1+\mu \sigma^{z}\right) \mathcal{U}_{\mathbf{k}+\mathbf{q}}^{\dagger} \sigma^{\beta} \sigma^{\alpha} \mathcal{U}_{\mathbf{k}+\mathbf{q}}\right] \\
& =\frac{1}{2} \sum_{\mu} f_{\mathbf{k}}^{\mu} \operatorname{Tr}\left[\left(1+\mu h_{\mathbf{k}}^{R}\right) \sigma^{\alpha} \sigma^{\beta}\right] \\
& -\frac{1}{2} \sum_{\mu} f_{\mathbf{k}+\mathbf{q}}^{\mu} \operatorname{Tr}\left[\left(1+\mu h_{\mathbf{k}+\mathbf{q}}^{R}\right) \sigma^{\beta} \sigma^{\alpha}\right]
\end{aligned}
$$

or, more explicitly,

$$
\begin{aligned}
& \mathcal{B}_{\mathbf{k q}}^{\alpha \alpha}=\sum_{\mu}\left(f_{\mathbf{k}}^{\mu}-f_{\mathbf{k}+\mathbf{q}}^{\mu}\right) \\
& \mathcal{B}_{\mathbf{k q}}^{x 0}=\mathcal{B}_{\mathbf{k q}}^{0 x}=\sum_{\mu} \mu\left(f_{\mathbf{k}}^{\mu} \sin \phi_{\mathbf{k}}-f_{\mathbf{k}+\mathbf{q}}^{\mu} \sin \phi_{\mathbf{k}+\mathbf{q}}\right), \\
& \mathcal{B}_{\mathbf{k q}}^{y 0}=\mathcal{B}_{\mathbf{k q}}^{0 y}=-\sum_{\mu} \mu\left(f_{\mathbf{k}}^{\mu} \cos \phi_{\mathbf{k}}-f_{\mathbf{k}+\mathbf{q}}^{\mu} \cos \phi_{\mathbf{k}+\mathbf{q}}\right), \\
& \mathcal{B}_{\mathbf{k q}}^{x z}=-\mathcal{B}_{\mathbf{k q}}^{z x}=i \sum_{\mu} \mu\left(f_{\mathbf{k}}^{\mu} \cos \phi_{\mathbf{k}}+f_{\mathbf{k}+\mathbf{q}}^{\mu} \cos \phi_{\mathbf{k}+\mathbf{q}}\right), \\
& \mathcal{B}_{\mathbf{k q}}^{y z}=-\mathcal{B}_{\mathbf{k q}}^{z y}=i \sum_{\mu} \mu\left(f_{\mathbf{k}}^{\mu} \sin \phi_{\mathbf{k}}+f_{\mathbf{k}+\mathbf{q}}^{\mu} \sin \phi_{\mathbf{k}+\mathbf{q}}\right) \\
& \mathcal{B}_{\mathbf{k q}}^{0 z}=\mathcal{B}_{\mathbf{k q}}^{z 0}=\mathcal{B}_{\mathbf{k q}}^{x y}=\mathcal{B}_{\mathbf{k q}}^{y x}=0 .
\end{aligned}
$$

Combining (A7) and (A11), we derive the following matrix equation

$$
\mathcal{A}_{\mathrm{kq}} \rho_{\mathrm{kq}}=\frac{1}{\mathcal{V}} \mathcal{B}_{\mathrm{kq}} V_{\mathbf{q}}
$$

where the upper indices are omitted for brevity. Inverting the matrix $\mathcal{A}_{\mathbf{k q}}$ and summing over $\mathbf{k}$, we obtain $\rho_{\mathbf{q}} \equiv$ $\left\langle\hat{\rho}_{\mathbf{q}}\right\rangle=\chi V_{\mathbf{q}}$, where

$$
\chi=\frac{1}{\mathcal{V}} \sum_{\mathbf{k}} \mathcal{A}_{\mathrm{kq}}^{-1} \mathcal{B}_{\mathrm{kq}}
$$

is a density response matrix of the non-interacting system. After the straightforward calculation, we recover from (A17) the expression (77) for the components of $\chi$.

Let us now take into account electron-electron interaction

$$
H_{\mathrm{int}}=\frac{1}{2 \mathcal{V}} \sum_{\mathbf{p}^{\prime} \mathbf{k}^{\prime} \mathbf{q}^{\prime}} \sum_{\sigma s} v_{\mathbf{q}^{\prime}} c_{\mathbf{p}^{\prime}+\mathbf{q}^{\prime}, \sigma}^{\dagger} c_{\mathbf{k}^{\prime}-\mathbf{q}^{\prime}, s}^{\dagger} c_{\mathbf{k}^{\prime} s} c_{\mathbf{p}^{\prime} \sigma}
$$

In the mean field approximation we obtain

$$
\begin{gathered}
\left\langle\left[\hat{\rho}_{\mathbf{k q}}^{\alpha}, H_{\text {int }}\right]\right\rangle=\frac{1}{\mathcal{V}} \sum_{\mathbf{q}^{\prime}} v_{\mathbf{q}^{\prime}} \rho_{\mathbf{q}^{\prime}}^{0} \times \\
\times\left\{\left\langle c_{\mathbf{k}}^{\dagger} \sigma^{\alpha} c_{\mathbf{k}+\mathbf{q}-\mathbf{q}^{\prime}}\right\rangle-\left\langle c_{\mathbf{k}+\mathbf{q}^{\prime}}^{\dagger} \sigma^{\alpha} c_{\mathbf{k}+\mathbf{q}}\right\rangle\right\} \\
+\frac{1}{2 \mathcal{V}} \sum_{\mathbf{q}^{\prime}, \mathbf{p}^{\prime}, \beta} v_{\mathbf{q}^{\prime}}\left\{\left\langle c_{\mathbf{k}+\mathbf{q}^{\prime}}^{\dagger} \sigma^{\beta} c_{\mathbf{p}^{\prime}}\right\rangle\left\langle c_{\mathbf{p}^{\prime}-\mathbf{q}^{\prime}}^{\dagger} \sigma^{\beta} \sigma^{\alpha} c_{\mathbf{k}+\mathbf{q}}\right\rangle\right. \\
\left.-\left\langle c_{\mathbf{k}}^{\dagger} \sigma^{\alpha} \sigma^{\beta} c_{\mathbf{p}^{\prime}}\right\rangle\left\langle c_{\mathbf{p}^{\prime}+\mathbf{q}^{\prime}}^{\dagger} \sigma^{\beta} c_{\mathbf{k}+\mathbf{q}+\mathbf{q}^{\prime}}\right\rangle\right\} .
\end{gathered}
$$

The first sum in (A19) corresponds to the direct Coulomb term. We treat it further in the RPA picking the only term $\mathbf{q}^{\prime}=\mathbf{q}$ out of the whole sum. We obtain the following contribution

$$
\begin{aligned}
& \left\langle\left[\hat{\rho}_{\mathbf{k q}}^{\alpha}, H_{\text {int }}\right]\right\rangle^{\mathrm{RPA}}= \\
& =\frac{v_{\mathbf{q}} \rho_{\mathbf{q}}^{0}}{\mathcal{V}}\left\{\left\langle c_{\mathbf{k}}^{\dagger} \sigma^{\alpha} c_{\mathbf{k}}\right\rangle-\left\langle c_{\mathbf{k}+\mathbf{q}}^{\dagger} \sigma^{\alpha} c_{\mathbf{k}+\mathbf{q}}\right\rangle\right\}=\frac{v_{\mathbf{q}} \rho_{\mathbf{q}}^{0}}{\mathcal{V}} \mathcal{B}_{\mathbf{k q}}^{\alpha 0},
\end{aligned}
$$

which accounts for the effect of screening.

From the second sum in (A19) we can extract the exchange self-energy term and the Hubbard's exchange correction to the RPA.

A contribution associated with the self-energy is obtained from A19) after picking out the summands with $\mathbf{p}^{\prime}=\mathbf{k}+\mathbf{q}^{\prime}$ and $\mathbf{p}^{\prime}=\mathbf{k}+\mathbf{q}$, i.e.

$$
\begin{aligned}
& \left\langle\left[\hat{\rho}_{\mathbf{k q}}^{\alpha}, H_{\mathrm{int}}\right]\right\rangle^{\Sigma}= \\
& =\frac{1}{2 \mathcal{V}} \sum_{\mathbf{q}^{\prime}, \beta} v_{\mathbf{q}^{\prime}}\left\{\left\langle c_{\mathbf{k}+\mathbf{q}^{\prime}}^{\dagger} \sigma^{\beta} c_{\mathbf{k}+\mathbf{q}^{\prime}}\right\rangle\left\langle c_{\mathbf{k}}^{\dagger} \sigma^{\beta} \sigma^{\alpha} c_{\mathbf{k}+\mathbf{q}}\right\rangle\right. \\
& \left.\quad-\left\langle c_{\mathbf{k}}^{\dagger} \sigma^{\alpha} \sigma^{\beta} c_{\mathbf{k}+\mathbf{q}}\right\rangle\left\langle c_{\mathbf{k}+\mathbf{q}+\mathbf{q}^{\prime}}^{\dagger} \sigma^{\beta} c_{\mathbf{k}+\mathbf{q}+\mathbf{q}^{\prime}}\right\rangle\right\} \\
& =-\sum_{\beta}\left\{\Sigma_{\mathbf{k}}^{\beta}\left\langle c_{\mathbf{k}}^{\dagger} \sigma^{\beta} \sigma^{\alpha} c_{\mathbf{k}+\mathbf{q}}\right\rangle-\Sigma_{\mathbf{k}+\mathbf{q}}^{\beta}\left\langle c_{\mathbf{k}}^{\dagger} \sigma^{\alpha} \sigma^{\beta} c_{\mathbf{k}+\mathbf{q}}\right\rangle\right\},
\end{aligned}
$$

where

$$
\begin{aligned}
\Sigma_{\mathbf{k}}^{\beta} & =-\frac{1}{2 \mathcal{V}} \sum_{\mathbf{q}^{\prime}} v_{\mathbf{q}^{\prime}-\mathbf{k}} \rho_{\mathbf{q}^{\prime} 0}^{\beta} \\
& =-\frac{1}{4 \mathcal{V}} \sum_{\mathbf{q}^{\prime}, \mu^{\prime}} v_{\mathbf{q}^{\prime}-\mathbf{k}} f_{\mathbf{q}^{\prime}}^{\mu^{\prime}} \operatorname{Tr}\left[\left(1+\mu^{\prime} h_{\mathbf{q}^{\prime}}^{R}\right) \sigma^{\beta}\right] .
\end{aligned}
$$

The self-energy $\Sigma_{\mathbf{k}}^{\beta}$ modifies the single-particle Hamiltonian $E_{\mathbf{k}}^{\beta} \rightarrow E_{\mathbf{k}}^{\beta}+\Sigma_{\mathbf{k}}^{\beta}$. After diagonalization we obtain the renormalized eigenvalues $\epsilon_{\mathbf{k}}^{\mu} \rightarrow \epsilon_{\mathbf{k}}^{\mu}+\Sigma_{\mathbf{k}}^{\mu}$,

$$
\Sigma_{\mathbf{k}}^{\mu}=-\frac{1}{2 \mathcal{V}} \sum_{\mathbf{q}^{\prime}, \mu^{\prime}} v_{\mathbf{q}^{\prime}-\mathbf{k}} f_{\mathbf{q}^{\prime}}^{\mu^{\prime}}\left[1+\mu \mu^{\prime} \cos \left(\phi_{\mathbf{k}}-\phi_{\mathbf{q}^{\prime}}\right)\right],
$$

which correspond to the same eigenstates (3). Besides the shift of a chemical potential, the spectrum renormalization results in an effective value of the Rashba splitting, which has been previously studied in Ref. 35 . In our consideration we will, however, neglect this effect and discard the contribution A21). 
The Hubbard's exchange term is given by the summands in (A19) with $\mathbf{p}^{\prime}=\mathbf{k}+\mathbf{q}+\mathbf{q}^{\prime}$ and $\mathbf{p}^{\prime}=\mathbf{k}$, i.e.

$$
\begin{aligned}
& \left\langle\left[\hat{\rho}_{\mathbf{k q}}^{\alpha}, H_{\mathrm{int}}\right]\right\rangle^{\mathrm{Hub}}= \\
& =\frac{1}{2 \mathcal{V}} \sum_{\mathbf{q}^{\prime}, \beta} v_{\mathbf{q}^{\prime}}\left\{\left\langle c_{\mathbf{k}+\mathbf{q}^{\prime}}^{\dagger} \sigma^{\beta} c_{\mathbf{k}+\mathbf{q}+\mathbf{q}^{\prime}}\right\rangle\left\langle c_{\mathbf{k}+\mathbf{q}}^{\dagger} \sigma^{\beta} \sigma^{\alpha} c_{\mathbf{k}+\mathbf{q}}\right\rangle\right. \\
& \left.\quad-\left\langle c_{\mathbf{k}}^{\dagger} \sigma^{\alpha} \sigma^{\beta} c_{\mathbf{k}}\right\rangle\left\langle c_{\mathbf{k}+\mathbf{q}^{\prime}}^{\dagger} \sigma^{\beta} c_{\mathbf{k}+\mathbf{q}+\mathbf{q}^{\prime}}\right\rangle\right\} \\
& =-\frac{1}{2 \mathcal{V}} \sum_{\mathbf{k}^{\prime}, \beta} v_{\mathbf{k}^{\prime}-\mathbf{k}} \mathcal{B}_{\mathbf{k q}}^{\alpha \beta} \rho_{\mathbf{k}^{\prime} \mathbf{q}}^{\beta} \\
& \approx-\frac{\bar{v}_{\mathbf{q}}}{2 \mathcal{V}} \sum_{\mathbf{k}^{\prime}, \beta} \mathcal{B}_{\mathbf{k q}}^{\alpha \beta} \rho_{\mathbf{k}^{\prime} \mathbf{q}}^{\beta}=-\frac{\bar{v}_{\mathbf{q}}}{2 \mathcal{V}} \sum_{\beta} \mathcal{B}_{\mathbf{k q}}^{\alpha \beta} \rho_{\mathbf{q}}^{\beta},
\end{aligned}
$$

where $\bar{v}_{\mathbf{q}}$ is approximately regarded at small $q$ as a constant $\equiv 2 J=\frac{2 \pi e^{2}}{k_{F}}$.

Collecting the contributions (A20) and A24 and adding them to (A16), we obtain the equation

$$
\mathcal{A}_{\mathbf{k q}} \rho_{\mathbf{k q}}=\frac{1}{\mathcal{V}} \mathcal{B}_{\mathbf{k q}}\left(V_{\mathbf{q}}+F \rho_{\mathbf{q}}\right)
$$

where $F=\operatorname{diag}\left\{v_{\mathbf{q}}-J,-J,-J,-J\right\}$. Solving it, we find that $\rho_{\mathbf{q}}=\tilde{\chi} V_{\mathbf{q}}$, where

$$
\tilde{\chi}=(1-\chi F)^{-1} \chi
$$

is a density response matrix of the interacting system in the Hubbard's approximation.

\section{APPENDIX B: EXPLICIT EXPRESSIONS FOR THE FUNCTIONS $\chi^{(j)}$}

Let us list the functions $\check{f}_{i}^{(j)}, C_{i}^{(j)}$ and $\delta_{i}^{(j)}$ which determine the response functions $\chi^{(j)}$ in Eqs. (18) and (19):

$$
\begin{aligned}
\check{f}_{i}^{(1)} & =0, \quad C_{i}^{(1)}=\frac{v-\mu y}{2 v^{2} z} \\
\delta_{i}^{(1)} & =\frac{\left(z^{2}-\lambda w\right)-\mu y v}{(v-\mu y) z} ; \\
\check{f}_{i}^{(2)} & =\frac{\lambda(\mu-y)}{2 z}, C_{i}^{(2)}=\frac{\mu \lambda\left(\lambda w+\beta_{i} z v+\mu y v-z^{2}\right)}{2 v^{2} z^{2}} \\
\delta_{i}^{(2)} & =\frac{\left(\gamma_{i} v+\mu y\right) z}{\lambda w+\beta_{i} z v+\mu y v-z^{2}} \\
\check{f}_{i}^{(3)} & =\frac{y(y-\mu)}{2 z^{2}}, \quad C_{i}^{(3)}=\frac{v z-\mu y z+\beta_{i} \mu y v}{2 v^{2} z^{2}} \\
\delta_{i}^{(3)} & =\frac{z^{2}-\lambda w+\gamma_{i} \mu y v}{v z-\mu y z+\beta_{i} \mu y v} \\
\check{f}_{i}^{(4)} & =0, \quad C_{i}^{(4)}=\frac{v+\mu y}{2 v^{2} z} \\
\delta_{i}^{(4)} & =\frac{z^{2}-\lambda w}{(v+\mu y) z} ; \\
\check{f}_{i}^{(5)} & =\frac{\mu-y}{2 z}, \quad C_{i}^{(5)}=-\frac{\mu\left(\lambda w+\beta_{i} z v-z^{2}\right)}{2 v^{2} z^{2}} \\
\delta_{i}^{(5)} & =\frac{\left(\gamma_{i} v-\mu y\right) z}{\lambda w+\beta_{i} z v-z^{2}} .
\end{aligned}
$$

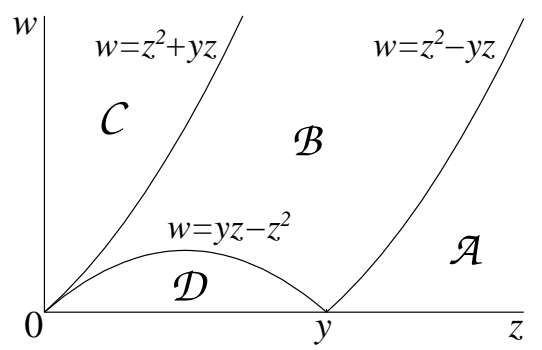

FIG. 9: The domains $\mathcal{A}, \mathcal{B}, \mathcal{C}$, and $\mathcal{D}$ corresponding to the different orderings (B6)-(B9) of the roots $\tau_{k}(25)$.

Employing (B1)-(B5) in the framework of the computational scheme elaborated in Ref. 23, one can derive Eqs. (23) and (24).

The actual limits of integration in the latter expressions require further detailing, since the corresponding integrands $\mathcal{L}^{(j)}(\tau)$ (29) and $\mathcal{R}^{(j)}(\tau)$ (30) are defined at $P(\tau)>0$ and $P(\tau)<0$, respectively. The polynomial $P(\tau)$ (31) has the roots $\tau_{k}$ (25), which can be ordered differently depending on the values of $w, z$, and $y$.

We identify the domains $\mathcal{A}, \mathcal{B}, \mathcal{C}$, and $\mathcal{D}$ in the plane $(z, w)$ [see Fig. 9] such that

$$
\begin{array}{cl}
\mathcal{A}: & \tau_{4}<\tau_{2}<\tau_{1}<\tau_{3}, \\
\mathcal{B}: & \tau_{4}<\tau_{2}<\tau_{3}<\tau_{1}, \\
\mathcal{C}: & \tau_{2}<\tau_{4}<\tau_{3}<\tau_{1}, \\
\mathcal{D}: & \tau_{4}<\tau_{3}<\tau_{2}<\tau_{1} .
\end{array}
$$

We also define the unit-step functions $\Theta(\mathcal{A})=\Theta\left(z^{2}-\right.$ $y z-w), \Theta(\mathcal{B})=\Theta\left(w-z^{2}+y z\right) \Theta\left(z^{2}+y z-w\right) \Theta(w+$ $\left.z^{2}-y z\right), \Theta(\mathcal{C})=\Theta\left(w-z^{2}-y z\right)$, and $\Theta(\mathcal{D})=\left(y z-z^{2}-\right.$ $w)$, which realize projections onto these domains. Using these definitions we make the following decomposition

$$
\begin{aligned}
\chi^{(j)} & =\check{\chi}^{(j)}+\Theta(\mathcal{A}) \chi_{a}^{(j)}+\Theta(\mathcal{B}) \chi_{b}^{(j)} \\
& +\Theta(\mathcal{C}) \chi_{c}^{(j)}+\Theta(\mathcal{D}) \chi_{d}^{(j)},
\end{aligned}
$$

where $\check{\chi}^{(j)}$ is nonzero only for $j=3$ and $j=5$ :

$$
\check{\chi}^{(3)}=-2 \nu y^{2} / z^{2}, \quad \check{\chi}^{(5)}=2 \nu y / z .
$$

Inside of each domain the root's ordering is fixed, and one has to find out how $\tau_{\sigma \lambda}(\ldots)$ occurring in Eqs. (23) and (24) are arranged among $\tau_{1}, \ldots, \tau_{4}$.

Let us introduce $x_{4}<x_{3}<x_{2}<x_{1}$, where $x_{k}$ 's are identified with $\tau_{k}$ 's differently in each domain according to (B6)-(B), and define the primitives for the imaginary

$$
\begin{aligned}
A_{1}^{(j)+}(x) & =\int_{x_{1}}^{x} d x^{\prime} \mathcal{L}^{(j)}\left(x^{\prime}\right), \quad x_{1}<x, \\
A_{2}^{(j)}(x) & =\int_{x_{3}}^{x} d x^{\prime} \mathcal{L}^{(j)}\left(x^{\prime}\right), \quad x_{3}<x<x_{2}, \\
A_{1}^{(j)-}(x) & =-\int_{x}^{x_{4}} d x^{\prime} \mathcal{L}^{(j)}\left(x^{\prime}\right), \quad x<x_{4},
\end{aligned}
$$


and the real parts

$$
\begin{aligned}
& B_{1}^{(j)}(x)=\int_{x_{2}}^{x} d x^{\prime} \mathcal{R}^{(j)}\left(x^{\prime}\right), \quad x_{2}<x<x_{1}, \\
& B_{2}^{(j)}(x)=\int_{x_{4}}^{x} d x^{\prime} \mathcal{R}^{(j)}\left(x^{\prime}\right), \quad x_{4}<x<x_{3} .
\end{aligned}
$$

They have to be further detailed in each domain as well. For example, in the domain $\mathcal{C}$ we have $x_{3}=\tau_{4} \equiv-z-y$, and therefore

$$
A_{2}^{(j)}(x) \rightarrow A_{2 c}^{(j)}(x)=\int_{-z-y}^{x} d x^{\prime} \mathcal{L}^{(j)}\left(x^{\prime}\right) .
$$

After such a specification the primitives (B12)-B16 can be explicitly found in terms of elliptic functions [see Appendix $\mathrm{C}$ of Ref. 23]. In particular, we quote the $A_{2 c}^{(j)}$ expressions for $j=2$ and $j=4$ :

$$
\begin{aligned}
A_{2 c}^{(2)}(x)=- & \frac{k_{c}\left[(w / z-y)^{2}-z^{2}\right]}{4 z w \sqrt{w}}\left\{2 w F\left(\varphi_{2 c}(x), k_{c}\right)\right. \\
& -(w / z)^{2} n_{2 c} \Pi\left(\varphi_{2 c}(x), n_{2 c}, k_{c}\right) \\
& \left.-\left(z^{2}-y^{2}\right) \widetilde{n}_{2 c} \Pi\left(\varphi_{2 c}(x), \widetilde{n}_{2 c}, k_{c}\right)\right\},(\mathrm{B} 18)
\end{aligned}
$$

$$
\begin{aligned}
A_{2 c}^{(4)}(x)= & \frac{w}{2\left(z^{2}-y^{2}\right) x} \sqrt{\frac{z x-w}{z x+w}\left[(x+y)^{2}-z^{2}\right]} \\
- & \frac{k_{c}\left((w / z-y)^{2}-z^{2}\right)}{4 \sqrt{w}\left(z^{2}-y^{2}\right)}\left\{z F\left(\varphi_{2 c}(x), k_{c}\right)\right. \\
& \left.-y \widetilde{n}_{2 c} \Pi\left(\varphi_{2 c}(x), \widetilde{n}_{2 c}, k_{c}\right)\right\} \\
+ & \frac{z \sqrt{w}}{k_{c}\left(z^{2}-y^{2}\right)} E\left(\varphi_{2 c}(x), k_{c}\right),
\end{aligned}
$$

where

$$
\begin{aligned}
\varphi_{2 c}(x) & =\arcsin \sqrt{\frac{(x+y+z)(w / z-y+z)}{2(z x+w)}}, \\
k_{c} & =\frac{2 \sqrt{w}}{\sqrt{(z+w / z)^{2}-y^{2}}} \\
n_{2 c} & =\frac{2 z}{w / z+z-y}, \quad \widetilde{n}_{2 c}=\frac{w}{z(z+y)} n_{2 c}
\end{aligned}
$$

Let us now establish the actual limits of integration in Eq. (23). Rewriting it in terms of (B12)-(B14), we obtain $\operatorname{Im} \chi^{(j)}$ in every domain

$$
\begin{aligned}
& -\frac{1}{\nu} \operatorname{Im} \chi_{a}^{(j)}=-\Theta\left(w / z-t_{1}\right)\left[A_{2 a}^{(j)}(w / z)-A_{2 a}^{(j)}\left(t_{1}\right)+A_{2 a}^{(j)}\left(-t_{1}\right)\right]-\Theta\left(t_{2}-z-y\right) A_{1 a}^{(j)-}\left(-t_{2}\right) \\
& +\Theta\left(t_{2}-z+y\right) A_{1 a}^{(j)+}\left(t_{2}\right)-\Theta(1-4 w)\left\{\Theta\left(t_{4}-z+y\right) A_{1 a}^{(j)+}\left(t_{4}\right)-\Theta\left(t_{3}-z+y\right) A_{1 a}^{(j)+}\left(t_{3}\right)\right. \\
& \left.-\Theta\left(w / z-t_{3}\right)\left[A_{2 a}^{(j)}(w / z)-A_{2 a}^{(j)}\left(t_{3}\right)+A_{2 a}^{(j)}\left(-t_{3}\right)\right]-\Theta\left(t_{4}-z-y\right) A_{1 a}^{(j)-}\left(-t_{4}\right)\right\}, \\
& -\frac{1}{\nu} \operatorname{Im} \chi_{b}^{(j)}=-\Theta\left(z-y-t_{1}\right)\left[A_{2 b}^{(j)}(z-y)-A_{2 b}^{(j)}\left(t_{1}\right)\right]-\Theta\left(z-y+t_{1}\right) A_{2 b}^{(j)}\left(-t_{1}\right) \\
& -\Theta\left(y-z-t_{1}\right) A_{2 b}^{(j)}(z-y)-\Theta\left(t_{2}-z-y\right) A_{1 b}^{(j)-}\left(-T_{2}\right)+A_{1 b}^{(j)+}\left(t_{2}\right)-\Theta(1-4 w) \times \\
& \times\left\{-\Theta\left(t_{4}-z-y\right) A_{1 b}^{(j)-}\left(-t_{4}\right)+\Theta\left(t_{4}-w / z\right) A_{1 b}^{(j)+}\left(t_{4}\right)-\Theta\left(t_{3}-w / z\right) A_{1 b}^{(j)+}\left(t_{3}\right)\right. \\
& -\Theta\left(z-y-T_{3}\right)\left[A_{2 b}^{(j)}(z-y)-A_{2 b}^{(j)}\left(t_{3}\right)\right]+\Theta\left(w / z-t_{3}\right) \Theta\left(z-y+t_{3}\right)\left[A_{2 b}^{(j)}(z-y)-A_{2 b}^{(j)}\left(-t_{3}\right)\right] \\
& \left.-\Theta\left(w / z-t_{4}\right)\left[A_{2 b}^{(j)}(z-y)-A_{2 b}^{(j)}\left(-t_{4}\right)\right]-\Theta\left(t_{4}-w / z\right) \Theta\left(w / z-t_{3}\right) A_{2 b}^{(j)}(z-y)\right\} \\
& -\frac{1}{\nu} \operatorname{Im} \chi_{c}^{(j)}=\Theta\left(t_{2}-w / z\right)\left[A_{1 c}^{(j)+}\left(t_{2}\right)-A_{1 c}^{(j)-}\left(-t_{2}\right)\right]-\Theta\left(z-y-t_{1}\right)\left[A_{2 c}^{(j)}(z-y)-A_{2 c}^{(j)}\left(t_{1}\right)\right] \\
& -\Theta\left(z-y+t_{1}\right) \Theta\left(z+y-t_{1}\right) A_{2 c}^{(j)}\left(-t_{1}\right)-\Theta\left(y-z-t_{1}\right) A_{2 c}^{(j)}(z-y)-\Theta(1-4 w) \times \\
& \times\left\{\Theta\left(t_{4}-w / z\right)\left[A_{1 c}^{(j)+}\left(t_{4}\right)-A_{1 c}^{(j)-}\left(-t_{4}\right)\right]-\Theta\left(z-y-t_{3}\right)\left[A_{2 c}^{(j)}(z-y)-A_{2 c}^{(j)}\left(t_{3}\right)\right]\right. \\
& +\Theta\left(z+y-t_{3}\right) \Theta\left(z-y+t_{3}\right)\left[A_{2 c}^{(j)}(z-y)-A_{2 c}^{(j)}\left(-t_{3}\right)\right]-\Theta\left(z+y-t_{4}\right) \Theta\left(z-y+t_{4}\right) \times \\
& \left.\times\left[A_{2 c}^{(j)}(z-y)-A_{2 c}^{(j)}\left(-t_{4}\right)\right]-\Theta\left(t_{4}-z-y\right) \Theta\left(z+y-t_{3}\right) A_{2 c}^{(j)}(z-y)\right\}, \\
& -\frac{1}{\nu} \operatorname{Im} \chi_{d}^{(j)}=A_{1 d}^{(j)+}\left(t_{2}\right)-A_{1 d}^{(j)-}\left(-t_{2}\right)+A_{1 d}^{(j)-}\left(-t_{4}\right)-A_{1 d}^{(j)+}\left(t_{4}\right) .
\end{aligned}
$$


In the above expressions we have used the definitions

$$
\begin{aligned}
& t_{1,2}=\frac{1}{2}[\sqrt{1+4 w} \mp 1], \\
& t_{3,4}=\frac{1}{2}[1 \mp \sqrt{1-4 w}] .
\end{aligned}
$$

The actual limits of integration for the real part (24) can be found in a similar way. However, Eq. (24) accounts only the term $\operatorname{Re} \chi^{(j), I}$. Complementing it by $\operatorname{Re} \chi^{(j), I I}$ [see the remark 5) in the end of Sec. III], we present the sum of the both terms in every domain

$$
\begin{aligned}
- & \frac{1}{\nu} \operatorname{Re} \chi_{a}^{(j)}=\Theta\left(t_{2}-z+y\right) B_{1 a}^{(j)}(z-y)+\Theta\left(z-y-t_{2}\right) B_{1 a}^{(j)}\left(t_{2}\right)+\Theta\left(t_{1}-w / z\right) B_{1 a}^{(j)}\left(t_{1}\right)-2 B_{1 a}^{(j)}\left(\tilde{t}_{1}\right) \\
& -\left[2+\Theta\left(w / z-t_{1}\right)\right] B_{2 a}^{(j)}(-w / z)-\Theta\left(t_{1}-w / z\right) B_{2 a}^{(j)}\left(-T_{1}\right)+2 B_{2 a}^{(j)}\left(-\tilde{t}_{2}\right)-\Theta\left(z+y-t_{2}\right) B_{2 a}^{(j)}\left(-t_{2}\right) \\
& +2 \Theta\left(y^{2}-4 w\right)\left[B_{2 a}^{(j)}\left(\tilde{t}_{3}\right)+B_{2 a}^{(j)}\left(\tilde{t}_{4}\right)\right]+2 \Theta\left(4 w-y^{2}\right)\left[B_{2 a}^{(j)}\left(\tilde{t}_{3}^{c}\right)+B_{2 a}^{(j)}\left(\tilde{t}_{4}^{c}\right)\right]+\Theta(1-4 w)\left\{\Theta\left(z-y-t_{4}\right) B_{1 a}^{(j)}\left(t_{4}\right)\right. \\
& +\Theta\left(t_{3}-w / z\right) \Theta\left(z-y-t_{3}\right) B_{1 a}^{(j)}\left(t_{3}\right)+\left[\Theta\left(t_{3}-z+y\right)+\Theta\left(t_{4}-z+y\right)\right] B_{1 a}^{(j)}(z-y)-\Theta\left(z+y-t_{4}\right) B_{2 a}^{(j)}\left(-t_{4}\right) \\
& \left.-\Theta\left(t_{3}-w / z\right) B_{2 a}^{(j)}\left(-t_{3}\right)-\Theta\left(w / z-t_{3}\right) B_{2 a}^{(j)}(-w / z)\right\}+\Theta(4 w-1)\left\{B_{1 a}^{(j)}\left(t_{3}^{c}\right)+B_{1 a}^{(j)}\left(t_{4}^{c}\right)-B_{2 a}^{(j)}\left(-t_{3}^{c}\right)\right. \\
& \left.-B_{2 a}^{(j)}\left(-t_{4}^{c}\right)\right\}+s_{1}^{(j)} \frac{\pi \sqrt{z^{2}-y^{2}}}{z}+\tilde{s}_{1}^{(j)} \frac{\pi w}{z \sqrt{z^{2}-y^{2}}}-2 s_{3}^{(j)}\left[B_{1 a}^{(j)}(z-y)-B_{2 a}^{(j)}(-w / z)\right]
\end{aligned}
$$

$-\frac{1}{\nu} \operatorname{Re} \chi_{b}^{(j)}=-B_{1 b}^{(j)}(w / z)+2 B_{1 b}^{(j)}\left(\tilde{t}_{1}\right)-\Theta\left(t_{1}-z+y\right) B_{1 b}^{(j)}\left(t_{1}\right)-\Theta\left(y-z-t_{1}\right) B_{1 b}^{(j)}\left(-t_{1}\right)-\Theta\left(z+y-t_{2}\right) B_{2 b}^{(j)}\left(-t_{2}\right)$

$+2 B_{2 b}^{(j)}\left(-\tilde{t}_{2}\right)-\Theta\left(w / z-t_{1}\right) B_{2 b}^{(j)}(-w / z)-\Theta\left(t_{1}-w / z\right) B_{2 b}^{(j)}\left(-t_{1}\right)-2 \Theta\left(y^{2}-4 w\right) \Theta\left(w-z^{2}\right)\left[B_{1 b}^{(j)}\left(\tilde{t}_{3}\right)+B_{1 b}^{(j)}\left(\tilde{t}_{4}\right)\right]$ $+2 \Theta\left(y^{2}-4 w\right) \Theta\left(z^{2}-w\right)\left[B_{2 b}^{(j)}\left(\tilde{t}_{3}\right)+B_{2 b}^{(j)}\left(\tilde{t}_{4}\right)-2 B_{2 b}^{(j)}(-w / z)\right]-2 \Theta\left(4 w-y^{2}\right)\left[B_{1 b}^{(j)}\left(\tilde{t}_{3}^{c}\right)+B_{1 b}^{(j)}\left(\tilde{t}_{4}^{c}\right)\right]$

$+\Theta(1-4 w)\left\{\left[\Theta\left(t_{3}-w / z\right)+\Theta\left(t_{4}-w / z\right)\right] B_{1 b}^{(j)}(w / z)+\Theta\left(w / z-t_{3}\right) \Theta\left(t_{3}-z+y\right) B_{1 b}^{(j)}\left(t_{3}\right)+\Theta\left(w / z-t_{4}\right) B_{1 b}^{(j)}\left(t_{4}\right)\right.$ $+\left[\Theta\left(t_{3}-w / z\right)+\Theta\left(t_{4}-w / z\right)\right] B_{2 b}^{(j)}(-w / z)-\Theta\left(z+y-t_{4}\right) \Theta\left(t_{4}-w / z\right) B_{2 b}^{(j)}\left(-t_{4}\right)+\Theta\left(y-z-t_{3}\right) B_{1 b}^{(j)}\left(-t_{3}\right)$ $\left.-\Theta\left(t_{3}-w / z\right) B_{2 b}^{(j)}\left(-t_{3}\right)\right\}+\Theta(4 w-1)\left\{B_{1 b}^{(j)}\left(t_{3}^{c}\right)+B_{1 b}^{(j)}\left(t_{4}^{c}\right)+B_{1 b}^{(j)}\left(-t_{3}^{c}\right)+B_{1 b}^{(j)}\left(-t_{4}^{c}\right)\right\}+\Theta(1-4 w) \Theta(y-z) \times$ $\times\left(s_{1}^{(j)} \frac{\pi w}{z^{2}}+\tilde{s}_{1}^{(j)} \pi-2 s_{2}^{(j)}\left[B_{1 b}^{(j)}(w / z)-B_{2 b}^{(j)}(-w / z)\right]\right)-\left[\Theta(1-4 w) \Theta\left(4 w-y^{2}\right)-\Theta\left(y^{2}-4 w\right)\right] \Theta(z-y) \times$ $\times\left(s_{1}^{(j)} \frac{\pi \sqrt{z^{2}-y^{2}}}{z}+\tilde{s}_{1}^{(j)} \frac{\pi w}{z \sqrt{z^{2}-y^{2}}}-2 s_{3}^{(j)}\left[B_{1 b}^{(j)}(w / z)-B_{2 b}^{(j)}(-w / z)\right]\right)$,

$-\frac{1}{\nu} \operatorname{Re} \chi_{c}^{(j)}=2 B_{1 c}^{(j)}\left(\tilde{t}_{1}\right)-\Theta\left(t_{2}-w / z\right) B_{1 c}^{(j)}(w / z)-\Theta\left(w / z-t_{2}\right) B_{1 c}^{(j)}\left(t_{2}\right)-\Theta\left(t_{1}-z+y\right) B_{1 c}^{(j)}\left(t_{1}\right)$

$-\Theta\left(y-z-t_{1}\right) B_{1 c}^{(j)}\left(-t_{1}\right)+\Theta\left(z+y-t_{1}\right) B_{2 c}^{(j)}(-z-y)+\Theta\left(t_{1}-z-y\right) B_{2 c}^{(j)}\left(-t_{1}\right)+\Theta\left(w / z-t_{2}\right) B_{2 c}^{(j)}\left(-t_{2}\right)$

$-2 B_{2 c}^{(j)}\left(-\tilde{t}_{2}\right)-2 \Theta\left(y^{2}-4 w\right)\left[B_{1 c}^{(j)}\left(\tilde{t}_{3}\right)+B_{1 c}^{(j)}\left(\tilde{t}_{4}\right)\right]-2 \Theta\left(4 w-y^{2}\right)\left[B_{1 c}^{(j)}\left(\tilde{t}_{3}^{c}\right)+B_{1 c}^{(j)}\left(\tilde{t}_{4}^{c}\right)\right]+\Theta(1-4 w) \times$ $\times\left\{\Theta\left(t_{4}-w / z\right) B_{1 c}^{(j)}(w / z)+\Theta\left(w / z-t_{4}\right) B_{1 c}^{(j)}\left(t_{4}\right)+\Theta\left(t_{3}-z+y\right) B_{1 c}^{(j)}\left(t_{3}\right)+\Theta\left(y-z-t_{3}\right) B_{1 c}^{(j)}\left(-t_{3}\right)\right.$ $+\left[\Theta\left(t_{4}-z-y\right)+\Theta\left(t_{3}-z-y\right)\right] B_{2 c}^{(j)}(-z-y)-\Theta\left(w / z-t_{4}\right) \Theta\left(t_{4}-z-y\right) B_{2 c}^{(j)}\left(-t_{4}\right)$ $\left.-\Theta\left(t_{3}-z-y\right) B_{2 c}^{(j)}\left(-t_{3}\right)\right\}+\Theta(4 w-1)\left\{B_{1 c}^{(j)}\left(t_{3}^{c}\right)+B_{1 c}^{(j)}\left(t_{4}^{c}\right)+B_{1 c}^{(j)}\left(-t_{3}^{c}\right)+B_{1 c}^{(j)}\left(-t_{4}^{c}\right)\right\}$ $+\Theta(1-4 w) \Theta(y-z)\left(s_{1}^{(j)} \frac{\pi w}{z^{2}}+\tilde{s}_{1}^{(j)} \pi-2 s_{2}^{(j)}\left[B_{1 c}^{(j)}(w / z)-B_{2 c}^{(j)}(-z-y)\right]\right)-\Theta(1-4 w) \Theta(z-y) \times$ $\times\left(s_{1}^{(j)} \frac{\pi \sqrt{z^{2}-y^{2}}}{z}+\tilde{s}_{1}^{(j)} \frac{\pi w}{z \sqrt{z^{2}-y^{2}}}-2 s_{3}^{(j)}\left[B_{1 c}^{(j)}(w / z)-B_{2 c}^{(j)}(-z-y)\right]\right)$,

$$
\begin{aligned}
- & \frac{1}{\nu} \operatorname{Re} \chi_{d}^{(j)}=2 B_{1 d}^{(j)}\left(\tilde{t}_{1}\right)-B_{1 d}^{(j)}\left(t_{1}\right)-B_{1 d}^{(j)}\left(-t_{1}\right)-2 B_{2 d}^{(j)}(z-y)+2 B_{2 d}^{(j)}\left(-\tilde{t}_{2}\right)-2 B_{1 d}^{(j)}\left(\tilde{t}_{4}\right) \\
& +B_{1 d}^{(j)}\left(t_{3}\right)+B_{1 d}^{(j)}\left(-t_{3}\right)+2 B_{2 d}^{(j)}\left(\tilde{t}_{3}\right)+s_{1}^{(j)} \frac{\pi w}{z^{2}}+\tilde{s}_{1}^{(j)} \pi-2 s_{2}^{(j)}\left[B_{1 d}^{(j)}(w / z)-B_{2 d}^{(j)}(z-y)\right] .
\end{aligned}
$$


In these expressions we have used along with B27 and $(\overline{B 28})$ the following arguments

$$
\begin{aligned}
\tilde{t}_{1,2} & =\frac{1}{2}\left[\sqrt{y^{2}+4 w} \mp y\right], \\
\tilde{t}_{3,4} & =\frac{1}{2}\left[-y \mp \sqrt{y^{2}-4 w}\right], \\
t_{3,4}^{c} & =\frac{1}{2}[1 \mp i \sqrt{4 w-1}], \\
\tilde{t}_{3,4}^{c} & =\frac{1}{2}\left[-y \mp i \sqrt{4 w-y^{2}}\right] .
\end{aligned}
$$

Note that $t_{3,4}^{c}$ and $\tilde{t}_{3,4}^{c}$ are complex-valued, which assumes the analytic continuation of elliptic functions hinted in Appendix B of Ref. 23 .

1 S. A. Wolf et al., Science 294, 1488 (2001).

2 I. Žutić, J. Fabian, and S. Das Sarma, Rev. Mod. Phys. 76, 323 (2004).

3 Y. A. Bychkov and E. I. Rashba, J. Phys. C 17, 6039 (1984).

4 J. Nitta, T. Akazaki, H. Takayanagi, and T. Enoki, Phys. Rev. Lett. 78, 1335 (1997).

5 T. Koga, J. Nitta, T. Akazaki, and H. Takayanagi, Phys. Rev. Lett. 89, 046801 (2002).

${ }^{6}$ E. G. Mishchenko and B. I. Halperin, Phys. Rev. B 68, 045317 (2003).

7 E. G. Mishchenko, A. V. Shytov, and B. I. Halperin, Phys. Rev. Lett. 93, 226602 (2004).

8 A. Burkov, A. S. Nunez, and A. H. MacDonald, Phys. Rev. B 70, 155308 (2004).

9 A. G. Mal'shukov, L. Y. Wang, C. S. Chu, and K. A. Chao, Phys. Rev. Lett. 95, 146601 (2005).

10 R. Raimondi, C. Gorini, P. Schwab, and M. Dzierzawa, Phys. Rev. B 74, 035340 (2006).

11 M. Pletyukhov, Phys. Rev. B 75, 155335 (2007).

12 A. Shnirman and I. Martin, Europhys. Lett. 78, 27001 (2007).

13 L.I. Magarill, A.V. Chaplik, and M.V. Éntin, JETP 92, 153 (2001).

14 E. Rashba, Phys. Rev. B 70, 161201(R) (2004).

15 S. I. Erlingsson, J. Schliemann, and D. Loss, Phys. Rev. B 71, 035319 (2005).

16 J. A. Maytorena, C. López-Bastidas, and F. Mireles, Phys. Rev. B 74, 235313 (2006).

17 A. Brataas, A. G. Mal'shukov, and K. A. Chao, Phys. Rev. B 56, R1684 (1997).
Transforming $\operatorname{Re} \chi^{(j), I I}$ into the representation in terms of $B_{1}^{(j)}$ and $B_{2}^{(j)}$, we accumulate the residue terms which are different for each response function. They are fully defined by the following sets of constants

$$
\begin{aligned}
s_{1}^{(1)} & =\tilde{s}_{1}^{(1)}=s_{2}^{(1)}=s_{3}^{(1)}=0, \\
s_{1}^{(2)} & =s_{2}^{(2)}=1, \quad \tilde{s}_{1}^{(2)}=s_{3}^{(2)}=0, \\
s_{2}^{(3,4)} & =s_{3}^{(3,4)}=1, \quad s_{1}^{(3,4)}=\tilde{s}_{1}^{(3,4)}=0 \\
\tilde{s}_{1}^{(5)} & =s_{2}^{(5)}=1, \quad s_{1}^{(5)}=s_{3}^{(5)}=0 .
\end{aligned}
$$

We recall once again that for $j=3$ and $j=5$ Eqs. (B29)-(B32) must be complemented by $\check{\chi}^{(j)}$ (B11).

18 C. A. Ullrich and M. E. Flatté, Phys. Rev. B 66, 205305 (2002).

19 W. Xu, Appl. Phys. Lett. 82, 724 (2003).

20 X. F. Wang, Phys. Rev. B 72, 085317 (2005).

21 G. Gumbs, Phys. Rev. B 72, 165351 (2005).

22 M. S. Kushwaha and S. E. Ulloa, Phys. Rev. B 73, 205306 (2006).

23 M. Pletyukhov and V. Gritsev, Phys. Rev. B 74, 045307 (2006).

24 F. Stern, Phys. Rev. Lett. 18, 546 (1967).

${ }^{25}$ H. Imamura, P. Bruno, and Y. Utsumi, Phys. Rev. B 69, 121303(R) (2004).

26 M. A. Ruderman and C. Kittel, Phys. Rev. 96, 99 (1954).

27 T. Kasuya, Prog. Theor. Phys. 16, 45 (1956).

28 K. Yosida, Phys. Rev. 106, 893 (1957).

29 G. D. Mahan, Many-Particle Physics (Plenum Press, New York, 1990).

30 I. S. Gradshteyn and I. M. Ryzhik, Table of Integrals, Series, and Products (Academic, New York, 1994).

31 V. M. Edelstein, Solid State Commun. 73, 233 (1990).

32 E. I. Rashba and Al. L. Efros, Appl. Phys. Lett. 83, 5295 (2003).

33 A. Shekhter, M. Khodas, and A. M. Finkel'stein, Phys. Rev. B 71, 165329 (2005).

34 D. C. Hamilton and A. L. McWhorter, in Light Scattering Spectra of Solids, edited by G. B. Wright (Springer, New York, 1969).

35 Guang-Hong Chen and M. E. Raikh, Phys. Rev. B 60, 4826 (1999). 